\title{
CMEs in the Heliosphere: II. A Statistical Analysis of the Kinematic Properties Derived from Single-Spacecraft Geometrical Modelling Techniques Applied to CMEs Detected in the Heliosphere from 2007 to 2017 by STEREO/HI-1
}

\author{
D. Barnes ${ }^{1}$ (D) - J.A. Davies ${ }^{1}(D)$ R.A. Harrison ${ }^{1}$ (D) J.P. Byrne ${ }^{1}$ - C.H. Perry ${ }^{1}$ (D)

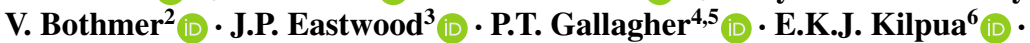 \\ C. Möstl ${ }^{7}(D) \cdot$ L. Rodriguez $^{8}(D) \cdot$ A.P. Rouillard ${ }^{9}$ D. Odstrčil ${ }^{10}(D)$
}

Received: 28 January 2019 / Accepted: 13 April 2019 / Published online: 14 May 2019

(C) The Author(s) 2019

\begin{abstract}
Recent observations with the Heliospheric Imagers (HIs) onboard the twin NASA Solar Terrestrial Relations Observatory (STEREO) spacecraft have provided unprecedented observations of a large number of coronal mass ejections (CMEs) in the inner heliosphere. In this article we discuss the generation of the HIGeoCAT CME catalogue and perform a statistical analysis of its events. The catalogue was generated as part of the EU FP7 HELCATS (Heliospheric Cataloguing, Analysis and Techniques Service) project (www.helcats-fp7.eu/). It is created by generating time/elongation maps for CMEs using observations from the inner (HI-1) and outer (HI-2) cameras along a position angle close to the CME apex. Next, we apply single-spacecraft geometric-fitting techniques to determine the kinematic properties of these CMEs, including their speeds, propagation directions, and launch times. The catalogue contains a total of 1455 events (801 from STEREO-A and 654 from STEREO-B) from April 2007 to the end of August 2017. We perform a statistical analysis of the properties of CMEs in HIGeoCAT and compare the results with those from the Large Angle Spectrometric Coronagraph (LASCO) CDAW catalogues (Yashiro et al. J. Geophys. Res. Space Phys.
\end{abstract}

$\triangle$ D. Barnes

david.barnes@stfc.ac.uk

1 STFC RAL Space, Rutherford Appleton Laboratory, Harwell Campus, Oxfordshire, OX11 0QX, $\mathrm{UK}$

2 Institute for Astrophysics, Georg August-University of Göttingen, 37077 Göttingen, Germany

3 Blackett Laboratory, Imperial College London, London, SW7 2AZ, UK

4 School of Physics, Trinity College Dublin, Dublin 2, Ireland

5 School of Cosmic Physics, Dublin Institute for Advanced Studies, Dublin 2, Ireland

6 Department of Physics, University of Helsinki, PO Box 64, 00014, Helsinki, Finland

7 Space Research Institute, Austrian Academy of Sciences, Schmiedlstrasse 6, 8042 Graz, Austria

8 Royal Observatory of Belgium, Ringlaan 3, 1180 Brussels, Belgium

9 Institut de Recherche en Astrophysique et Planetologie, 9 Ave du Colonel Roche, 31028 Toulouse Cedex 4, France

10 School of Physics, Astronomy and Computational Sciences, George Mason University, Fairfax, VA 22030-4444, USA 
109, A07105, 2004) and the COR-2 catalogue of Vourlidas et al. (Astrophys. J. 838, 141, 2004) during the same period. We find that the distributions of both speeds and latitudes for the HIGeoCAT CMEs correlate with the sunspot number over the solar cycle. We also find that the HI-derived CME speed distributions are generally consistent with coronagraph catalogues over the solar cycle, albeit with greater absolute speeds due to the differing methods with which each is derived.

Keywords Coronal mass ejections $\cdot$ Heliosphere $\cdot$ Space weather

\section{Introduction}

Coronal mass ejections (CMEs) are large magnetised clouds of plasma that originate from the Sun and propagate outward through interplanetary space (e.g. Webb and Howard, 2012). They are established to be the cause of the most severe geomagnetic disturbances experienced on Earth (e.g. Gosling et al., 1991; Kilpua et al., 2005; Zhang et al., 2007; Richardson and Cane, 2012; Kilpua et al., 2017); therefore understanding their propagation is vital in attempts to mitigate the potentially adverse effects of Earth-directed CMEs. There exist numerous statistical studies of CME properties, such as their frequency, size, and kinematics, dating back to the earliest remote-sensing observations during the 1970s. Historically, these studies have mainly been based on coronagraph imagery, and hence they were limited to within around $30 \mathrm{R}_{\odot}$ of the Sun. The more recent advent of heliospheric imaging has enabled such analysis to be applied to CMEs imaged in the heliosphere. This allows us to study their propagation to much larger distances and better understand the physical processes in action in interplanetary space, and it provides extremely useful observations for space-weather forecasting purposes.

By far the most comprehensive coverage of CMEs in time comes from the $\mathrm{C} 2$ and $\mathrm{C} 3$ coronagraphs ( $\mathrm{C} 1$ was lost shortly after the mission began) of the Large Angle and Spectrometric Coronagraph (LASCO: Brueckner et al., 1995) package onboard the Solar and Heliospheric Observatory (SOHO), which have been in operation since 1995 at the SunEarth Lagrange point $\mathrm{L}_{1}$. Prior to this, the first space-borne coronagraph was carried on the Orbiting Solar Observatory 7 (OSO 7, 1971 - 1974: Tousey, Howard, and Koomen, 1974), followed by another onboard Skylab in 1979 (Gosling et al., 1974). Further such instruments were carried on both the Solwind mission (1979 - 1885: Sheeley et al., 1980a) and the Solar Maximum Mission (SMM, 1980 - 1989: MacQueen et al., 1980). During the intermediate period from 1989 to 1995 there existed coverage from ground-based sources, such as the Mauna Loa MK3 (1980 - 1999: Fisher et al., 1981) and MK4 (1998-2013: Elmore et al., 2003) Coronameters; thus we are approaching almost four solar cycles of continuous CME observations. This has provided a means to study both the variation of CME parameters at coronal altitudes over a single cycle and how these variations have changed over the last four cycles with the observed decline in solar activity. Of course, since the launch of the Solar Terrestrial Relations Observatory (STEREO) in late 2006, LASCO observations have been complemented by visible-light coronal imagery from a vantage point off the Sun-Earth line from the two STEREO spacecraft. Each STEREO spacecraft hosts an EUV imager (EUVI: e.g. Aschwanden et al., 2014), two coronagraphs (COR-1 and -2: Howard et al., 2008), and two wide-angle $\mathrm{HI}$ instruments (HI-1 and -2: Eyles et al., 2009).

Hundhausen et al. (1984) compared CME observations from the coronagraph onboard Skylab (Sheeley et al., 1980b) in 1973-1974 (solar minimum) with those from SMM at solar maximum in 1980. The results showed an increased CME rate at solar maximum, 
albeit only by $\approx 20 \%$ and a broader latitudinal distribution; $\approx 73 \%$ of CMEs in the solarminimum data had central position angles (PAs) within $45^{\circ}$ of the Equator compared with a uniform PA-distribution seen at solar maximum. An early study by Howard et al. (1985) established many general CME properties, based on 998 CMEs in Solwind observations during 1979-1981, which corresponded to solar maximum of Cycle 21. They determined a mean plane-of-sky (POS) speed of $470 \mathrm{~km} \mathrm{~s}^{-1}$ and an average span of $45^{\circ}$, centred very broadly on the Equator. The total rate of CMEs was found to be $1.8 \mathrm{day}^{-1}$ for all events and 0.9 day $^{-1}$ for "major" CMEs. Burkepile and St. Cyr (1993) produced a catalogue of 1569 CMEs over the years 1980 and $1984-1989$ using imagery from the SMM coronagraph. The authors found a similar solar-cycle variation to that shown by Hundhausen et al. (1984). A broad PA-distribution was observed during the maximum of Solar Cycle 21 (1980), but that distribution narrowed around the Equator toward solar minimum in 1986. Over the ascending phase of Cycle 22 (1989), the distribution again broadened. During the latter period the CME-distribution remained centred around the Equator, and the mean CME width was approximately $46^{\circ}$, similar to the solar-maximum value $\left(45^{\circ}\right)$ determined by Howard et al. (1985). The POS CME speeds derived by Burkepile and St. Cyr (1993) did not vary significantly as a function of solar cycle, with an overall mean (median) value of $349\left(285 \mathrm{~km} \mathrm{~s}^{-1}\right)$. The frequency of CMEs was, however, found to vary significantly over the solar cycle, with a peak of 1.6 day $^{-1}$ on average in 1989 and a minimum of 0.2 day $^{-1}$ in 1985. St. Cyr et al. (1999) compared the results of a study of CMEs observed from the ground by MK3, during $1980-1989$, to those from Solwind and SMM. The authors found the CME properties from all three data sets to be relatively consistent: the mean (median) speed from MK3 was 390 $\left(309 \mathrm{~km} \mathrm{~s}^{-1}\right)$ and the mean (median) angular width was $37\left(33^{\circ}\right)$, with CMEs tending to emerge near the Equator. St. Cyr et al. (1999) found the same correlation between CME frequency and sunspot number (SSN) for all three instruments; however, the total number of CMEs imaged by MK3 (141) was much lower due to the difficulty that is introduced by scattered light in the sky when observing from the ground. In a recent follow-up article (St. Cyr et al., 2015) based on MK3 observations during the period 1989-1996, the peak and decline of Solar Cycle 22 showed that the strong correlation between SSN and CME rate continued throughout this period; overall speed, width, and latitude distributions were also consistent with previous results. Of the 141 MK3 CMEs studied by St. Cyr et al. (1999), the authors were able to associate 131 with features seen by SMM; the POS fields-of-view (FOVs) of these instruments span 1.1-2.4 $\mathrm{R}_{\odot}$ and 1.5-6 $\mathrm{R}_{\odot}$, respectively, providing a means for studying near-Sun CME acceleration. Over this range the authors found a mean (median) acceleration of $264\left(44 \mathrm{~ms}^{-2}\right)$, with the acceleration being positive for $87 \%$ of events, showing that the forces driving CME acceleration dominate throughout this altitude regime.

With the launch of SOHO in December 1995, LASCO has provided near-continuous coverage of CMEs over the whole of Solar Cycle 23 and the majority of the current cycle, 24. The LASCO instruments have a combined range from 1.1 to $32 \mathrm{R}_{\odot}\left(1.5\right.$ to $32 \mathrm{R}_{\odot}$ after the loss of the inner C1 coronagraph in 1998), a far greater distance than was covered by the instruments discussed previously. A comprehensive list of LASCO-observed CMEs is contained within the so-called CDAW (Coordinated Data Analyses Workshop) LASCO event list (Yashiro et al., 2004). In that article, the authors also present a statistical analysis of nearly 7000 LASCO CMEs observed from 1996 to 2002, corresponding to the maximum of Solar Cycle 23. They showed a monotonic increase in mean CME angular width from $47^{\circ}$ at minimum (1996) up to $61^{\circ}$ at early maximum (1999) and a decrease to $53^{\circ}$ at late maximum (2002). A similar trend is seen with CME speeds, where the mean value increases from approximately 300 to $500 \mathrm{~km} \mathrm{~s}^{-1}$ between minimum and maximum. CME acceleration is typically described as occurring under two regimes (e.g. Zhang et al., 2004; Zhang 
and Dere, 2006); the initiation phase occurs in the low corona $\left(<2 \mathrm{R}_{\odot}\right)$ and the propagation phase occurs above this in the outer corona and heliosphere. The latter is determined by the mutual Lorentz, gravitational, and drag forces acting on the CME. The Lorentz force dominates up to a few solar radii, whilst aerodynamic drag dominates beyond this (Sachdeva et al., 2015, 2017). Beyond a few solar radii, CME speeds are typically found to remain relatively constant; however, the very slowest events are seen to accelerate well into the LASCO FOV and the very fastest to decelerate (Yashiro et al., 2004; Gopalswamy et al., 2009). This is evidence of the drag forces from the ambient solar wind acting on CMEs and causing their speeds to tend toward its value of around 400 to $500 \mathrm{~km} \mathrm{~s}^{-1}$. Much recent analysis of the continuing stream of LASCO-C2 and -C3 data has focused on the differences in CME properties between the weak Solar Cycle 24 and the previous three stronger cycles. Interestingly, the analysis by Gopalswamy et al. (2014a,b, 2015) show that the CME rate did not drop in Cycle 24, despite a 40\% decrease in SSN, and they also demonstrate a large increase in the abundance of both wide CMEs and high-latitude CMEs since the polar-field reversal of Cycle 23, in 2004. Those authors suggest that this is due to the weakened polar magnetic-field strength of Cycle 24, which serves to reduce both the ambient magnetic and dynamic solar-wind pressure and therefore allows CMEs to expand more easily. The recent development of wide-angle imagers - firstly the Solar Mass Ejection Imager (SMEI: Eyles et al., 2003) onboard Coriolis, followed by the STEREO/Heliospheric Imagers (HIs: Eyles et al., 2009) - extends the range of observations of CMEs well beyond those of the aforementioned coronagraphs. Indeed, the HI instruments have permitted the tracking of CMEs to $1 \mathrm{AU}$ and beyond (e.g. Byrne et al., 2010; Davis, Kennedy, and Davies, 2010; Möstl et al., 2010; Savani et al., 2012; Harrison et al., 2012; Rollett et al., 2014; Temmer et al., 2014). A substantial increase in the number of CMEs catalogued and analysed based on HI data was achieved during the three-year EU-funded Heliospheric Cataloguing Analysis and Techniques Service (HELCATS) project, which ran from May 2014. The core output of this project is the HICAT catalogue, consisting of detections of CMEs by the inner (HI-1) camera on the twin NASA STEREO spacecraft. This catalogue contains basic, manually identified observational properties of the CMEs, such as their PA extent in HI, time of entry into the HI FOV, and the observing spacecraft (A or B). Version 5 of HICAT (doi:10.6084/m9.figshare.5803152) extends from April 2007 until August 2017 and contains 2059 detections. The generation of this catalogue is described by Harrison et al. (2018), hereafter referred to as Article 1. The reader is encouraged to refer to Article 1 for a thorough introduction to the HICAT catalogue, not least because further analysis of the majority of the HICAT CMEs forms the basis of the generation of the HIGeoCAT catalogue of CME kinematic properties described in this article. Like HICAT, HIGeoCAT was also generated under HELCATS.

As is evident from the discussion above, there are two methods by which CME catalogues are produced: by "manual" human inspection of images or by automatic identification using pattern-recognition algorithms. Manual catalogues include the CDAW catalogue (Yashiro et al., 2004), produced using LASCO data, the SMEI catalogue of Webb et al. (2006), and, more recently, the Citizen Science HI catalogue (Barnard et al., 2014). Likewise, CACTus (Computer Aided CME Tracking; LASCO and COR: Robbrecht, Berghmans, and Van der Linden, 2009), CORIMP (Coronal Image Processing; LASCO and COR: Byrne et al., 2012; Morgan, Byrne, and Habbal, 2012), and SEEDS (Solar Eruptive Event Detection System; LASCO: Olmedo et al., 2008) represent catalogues produced by automatic means. Some catalogues, such as the COR-2 catalogue of Vourlidas et al. (2017), are produced by a combination of both manual and automatic detections. Although the above list is far from comprehensive, it does reveal a certain tendency for the heliospheric-imaging community to opt 
for manual techniques, whilst automatic identification methods are more commonly applied to coronagraph observations. Tappin et al. (2012) apply an automatic-detection algorithm, Automatic CME Detection tool (AICMED), to SMEI data and conclude that, at best, it may be used as a "region of interest highlighter" due to a high false-alarm rate caused by the difficulty of identifying faint CMEs in wide-angle imagers. This is less of issue in coronagraph data, where the K-corona dominates over dust-scattered light, and so automatic-detection methods have enjoyed much more success. Further difficulties of CME detection in HI are caused by the jitter experienced by STEREO-B, which is the result of vibrations caused by the attitude-control reaction wheels. Kirnosov, Chang, and Pulkkinen (2016), however, combine COR-2 and HI-1 observations to automatically detect CME leading edges in the imagery, and CACTusCAT, which automatically detects CMEs in HI, was produced as part of HELCATS (Pant et al., 2016). The merits and difficulties of the manual versus automaticdetection methods are tackled directly by Yashiro, Michalek, and Gopalswamy (2008), who compare the CDAW and CACTus data sets. They showed that the manual CDAW catalogue had missed a large number of narrow CMEs detected by CACTus, but CACTus was seen to greatly overestimate the number of fast $\left(\geq 1000 \mathrm{~km} \mathrm{~s}^{-1}\right)$ events. One reason for this is that small events are very numerous and must be left out of manual-identification catalogues for the practical reason that recording them is too time-consuming. Automatic detection is beneficial because it avoids the time and tedium of identifying events by eye, particularly for large data sets. It is also objective in that it must be based on definite rules. However, the determination of kinematic properties in coronagraph data often relies on improper assumptions that can be circumvented when using data from heliospheric imagers. Specifically, a CME observed in a coronagraph is assumed to be travelling in the sky plane; that is, the plane intersecting the Sun centre, perpendicular to the Sun-Earth line. The basis behind this is that the nature of Thomson scattering causes CMEs in the sky plane to appear brighter than those away from it (Minnaert, 1930; van de Hulst, 1950; Tappin and Howard, 2009). As, for example, Gopalswamy et al. (2009) acknowledge, a consequence of this is that the true speeds of CMEs that are propagating out of the sky plane will be underestimated in coronagraph catalogues due to projection effects. A typical method for tracking CMEs in HI is to assume that they have reached a constant radial velocity by the time that they enter the FOV, and travel at a constant speed thereafter. This constant radial speed is manifested as an apparent angular acceleration in elongation, which is a function of CME speed and direction (Sheeley et al., 2008). These two parameters, and also the CME launch time, can therefore be estimated by tracking the observed CME elongation angle, as a function of time, through the combined HI-1 and HI-2 FOV, and performing a multi-variate fit (see Section 2).

The work presented in this article uses the HIGeoCAT catalogue that builds upon the HICAT CME catalogue (described above) as part of HELCATS Work Package 3 (WP3: www.helcats-fp7.eu/catalogues/wp3_cat.html). In WP3, kinematic properties of HI-observed CMEs are derived using a number of different methods, including single-spacecraft geometrical-fitting techniques. We take a large subset of the HICAT CMEs and track them through the combined HI-1 and HI-2 FOVs in order to record their observed elongation as a function of time. We are unable to do this for the full set of HICAT CMEs for several reasons, the most significant reason being that many of the HICAT events were small, faint CMEs that cannot be tracked for a sufficiently long time in order to apply the geometrical models to them. Other reasons include occasional data gaps in HI or because CMEs propagate at position angles too far from the Ecliptic to be tracked. Several different single-spacecraft geometrical models, based on assumptions of CME morphology and motion (Davies et al., 2012), are then applied to the time-elongation profiles of the remaining subset in order to infer kinematic properties, more specifically speed, direction, and 
launch time. The results of this form a new catalogue, which we call HIGeoCAT. Following September 2014 both spacecraft entered a period of reduced communications as they approached superior conjunction with the Sun and contact with STEREO-B was lost entirely. Since November 2015, STEREO-A has resumed normal operations and so the total range of observations covered here (April 2007 -August 2017) spans the 11 years covering the decline of Solar Cycle 23 and the majority of Cycle 24. The 2017 limit is chosen for consistency with Article 1, however, at the time of writing we are continuing to receive data from STEREO-A.

In this article we begin by describing in detail the means by which the HIGeoCAT CME catalogue is generated from HI data. This is followed by a statistical analysis of the resulting kinematic properties of CMEs in the catalogue. The solar-cycle behaviour of CME speeds, propagation directions, and launch times are then compared to existing coronagraph catalogues from LASCO (Yashiro et al., 2004; Vourlidas et al., 2017).

\section{HELCATS Heliospheric Imager Geometrical Catalogue (HIGeoCAT)}

\subsection{Geometric Modelling Techniques and CME Tracking}

The work here builds upon that carried out in Article 1 by taking a subset of those CMEs identified in HICAT and applying three single-spacecraft geometric fitting techniques to determine their kinematic properties. These techniques are summarised by Davies et al. (2012). The techniques apply the assumptions that the speed of the CME remains constant over the extent of the time-elongation profile FOV and that it possesses a predetermined morphology, in terms of angular extent. We track each CME at a single, fixed PA, as close to the CME apex as is practicable, and therefore these assumptions in fact apply to the morphology of the CME cross-section in the plane defined by that PA. The PA is chosen to correspond to the most advanced part of the CME; however, for very faint CMEs we are forced to use the part that is most visible. The first technique (fixed $\phi$ or FP: Kahler and Webb, 2007; Rouillard et al., 2008; Sheeley et al., 2008), assumes the CME to be a radially propagating point source, whilst the second (harmonic mean or HM: Lugaz, Vourlidas, and Roussev, 2009; Lugaz, 2010) assumes it to be represented by a radially propagating, expanding circle anchored to the Sun. In the third, and final, model (self-similar expansion or SSE) the CME is expressed as an expanding circle, which propagates radially but with a fixed half-width $[\lambda]$. In this implementation we use $\lambda=30^{\circ}$, and the FP and HM models are in fact extreme cases of the SSE with respective values of $\lambda=0^{\circ}$ and $90^{\circ}$ (Davies et al., 2012; Möstl and Davies, 2013). All three may be described by the SSE equation

$$
R=v\left(t-t_{0}\right)=\frac{r_{0} \sin (\epsilon(t))(1+\sin (\lambda))}{\sin (\epsilon(t)+\phi)+\sin (\lambda)}
$$

where $v$ is CME apex speed, $t$ is time, $t_{0}$ is launch time, $r_{0}$ is Sun-spacecraft distance, $\epsilon$ is the elongation, and $\phi$ is the spacecraft-Sun-CME apex angle. The FP, SSE, and HM models assume respective half-widths of $\lambda=0^{\circ}, 30^{\circ}$, and $90^{\circ}$. Figure 1 shows an example of the propagation of the same CME through the HI-1 FOVs of both STEREO-A and -B. Although, in this case, the CME is observed by both STEREO-A and STEREO-B, this is not true of all CMEs. The purpose of the work in this article is to apply single-spacecraft observations to CMEs and as such CMEs that are observed by both STEREO spacecraft appear as two separate entries in both HICAT and HIGeoCAT. In fact, a follow-up article will discuss the results 

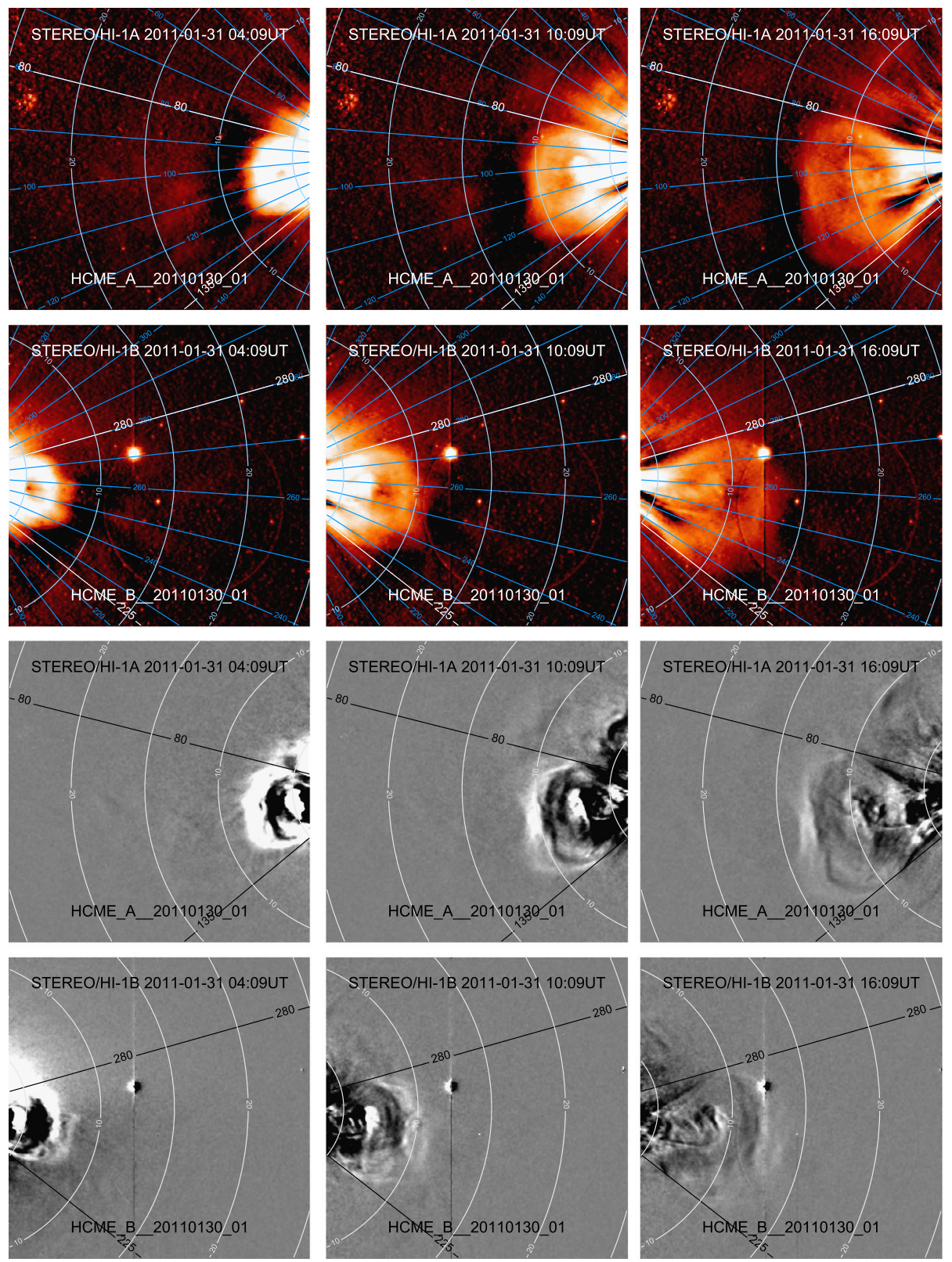

Figure 1 Example of HI-1 images of a CME observed by both STEREO spacecraft. The top row are background subtracted images are from HI-1A and are taken 6,12, and 18 hours after the CME entered the HI-1A FOV. The second row shows the corresponding background subtracted images taken from HI-1B at the same times. The equivalent running-difference images from rows one and two are shown in rows three and four. The position angle extent of the CME has been over-plotted in each case. 
of the application of analogous stereoscopic modelling techniques (Davies et al., 2013) to CMEs tracked through the FOVs of both STEREO spacecraft. The CME in Figure 1 appears in HIGeoCAT as both HCME_A_20110130_01 and HCME_B_20110130_01, which uses the same code as HICAT to identify CMEs. For each suitable CME in HICAT we produce a running-difference time-elongation map (Sheeley et al., 2008) at a fixed position angle using a combination of HI-1 and HI-2 data from the observing spacecraft. This corresponds to an elongation range of approximately $4^{\circ}$ to $88^{\circ}$ at a PA in the Ecliptic, which becomes less at PAs above and below this. We select the PA that is as close to the CME apex as is practicable. In the cases of very faint CMEs we instead chose the PA at which the CME can be most clearly seen and which is close to its apex. We then track the time-elongation profile of the CME leading edge as a function of time by manually selecting points on the time-elongation map. Using running-differenced HI images we typically see the leading edge of a CME as a white edge followed by a sharp white-black boundary (Figure 2, for example). Therefore, we define the CME leading edge as the first white edge that is visible. The points selected are used to constrain a fitting algorithm, for each of the three models, assuming that $v$ and $\phi$ remain constant in each case. This is achieved by using Equation 1 to estimate the three parameters $v, \phi$, and $t_{0}$ by means of a curve-fitting method similar to that of Savani et al. (2009) and Möstl et al. (2010). This is a non-linear optimisation based on the downhill-simplex method (Nelder and Mead, 1965). Here, we instead use the MPFIT routines of Markwardt (2009) based on the Levenberg-Marquardt algorithm (Moré, 1978). This is an iterative algorithm, whereby the values of the three free parameters $[v$, $\phi$, and $t_{0}$ ] are located by minimising the residual between theoretical and observed tracks. In our implementation, constraints are placed on each parameter; $v$ must lie in the range $1-5000 \mathrm{~km} \mathrm{~s}^{-1}, t_{0}$ must lie within ten days of the innermost point, and $\phi$ must be a value that corresponds to a CME within the FOV. This range is $0-180^{\circ}$ for a FP CME, however, this increases for the more complex geometries introduced by setting $\lambda \neq 0^{\circ}$. Finally, the tracking and process is repeated five times for each CME in an attempt to reduce random error. Afterwards, we apply the fitting process to each of these five data sets to derive five sets of fitted parameters. We record the mean value of each parameter.

Figure 2 shows the HI time-elongation data, from both STEREO-A and -B, for the CME in Figure 1, where the position angle used for each spacecraft is shown at the top of the figure. Panels a and b present time-elongation maps (often called J-maps) produced using running-difference HI-1 and HI-2 images. Within a time-elongation map, a propagating density structure (such as a CME front) is manifested as an inclined track that transitions between bright and dark. This corresponds to the increase and subsequent reduction in density, allowing it to easily be tracked. In both the STEREO-A and -B J-maps in Figure 2, the $\mathrm{CME}$ can be manually tracked out to between 30 and $40^{\circ}$, after which it becomes too faint to distinguish from the background. This is due to the reduction in density, and hence in brightness, as the CME propagates away from the Sun and makes it difficult to track all but the very brightest events far into the HI-2 FOV. The J-maps in Figure 2 both correspond to PAs close to the Ecliptic, where there is continuous elongation coverage between the HI-1 and HI-2 FOV. This is not the case for PAs further from the Ecliptic, as illustrated by the dashed and dotted lines in Figure 3; a gap in elongation is present between the two FOVs. We exclude from our analysis CMEs that suffer from large gaps in their time-elongation profiles, due to their PA or missing data (or corrupted images). Neither do we track CMEs that were classified as poor in HICAT (Harrison et al., 2018). The definition of poor, fair, and good CMEs is covered both in Article 1 and below. Panels e and $\mathrm{f}$ in Figure 2 show the tracked time-elongation profiles of each $\mathrm{CME}$ with the $\lambda=30^{\circ} \mathrm{SSE}$ fitted parameters printed and their theoretical tracks over-plotted (solid line). The CME used as an example 

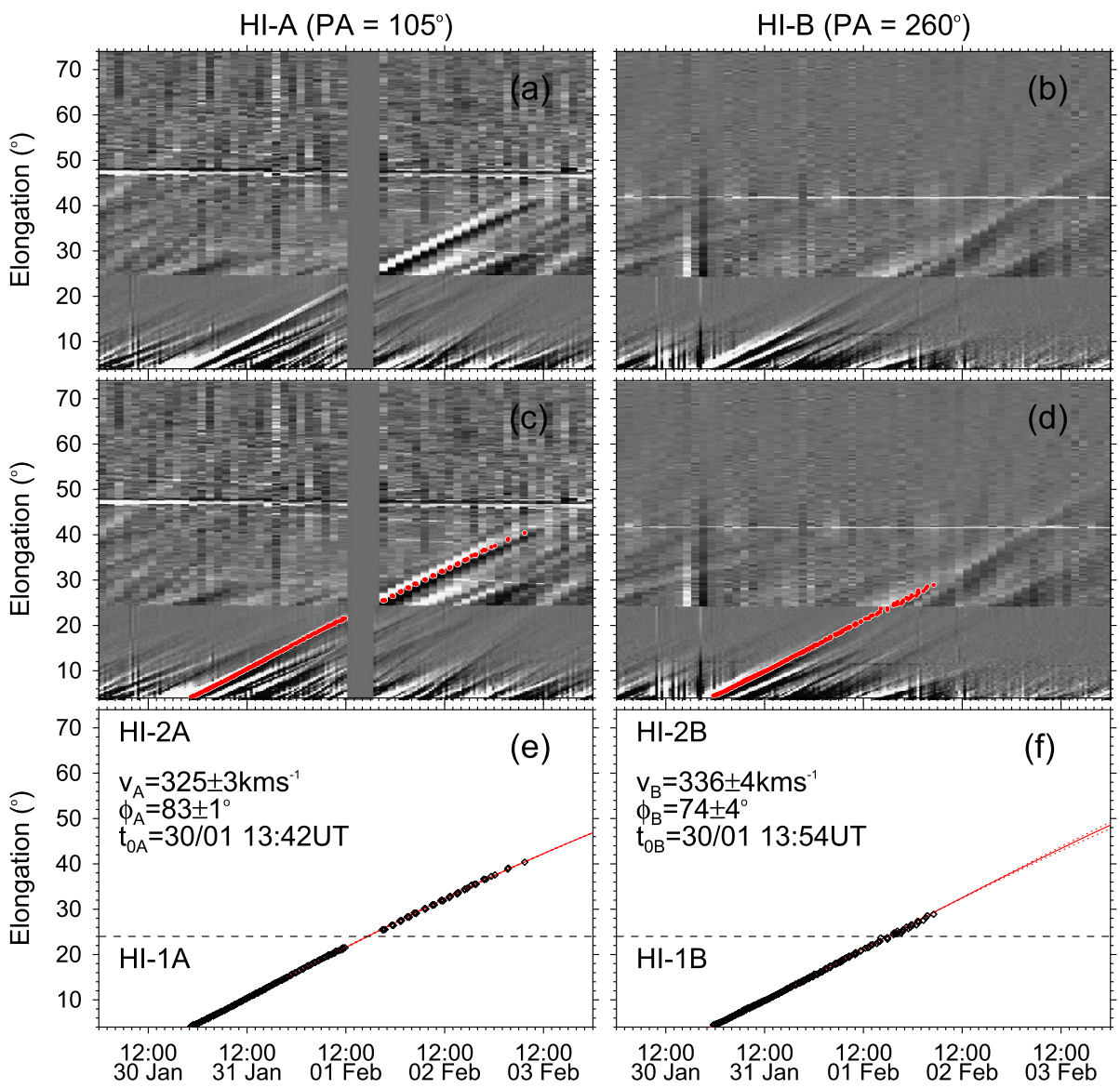

Figure 2 Time-elongation data for the HIGeoCAT CMEs HCME_A_20110130_01 and HCME_B_ 20110130_01 first observed by HI-1A and HI-1B on 30 January 2011 (Figure 1). Panels a and b show HI running-difference time-elongation maps for STEREO-A and STEREO-B, respectively, constructed along a position angle indicated by PA. The same data are shown in panels $\mathbf{c}$ and $\mathbf{d}$ where the over-plotted red circles show the manually tracked time-elongation profile of a CME in each. Panels $\mathbf{e}$ and $\mathbf{f}$ show the same fitted time-elongation profiles, this time with the best-fit parameters (from the SSE model, $\lambda=30^{\circ}$ ) printed and a red line representing the corresponding theoretical time-elongation profile of the CME. The gap in the top-left J-map corresponds to an eight-hour period, beginning at around 12:00 UT on 01 February, where there are no data from either HI instrument on STEREO-A.

in both Figures 1 and 2 has been chosen to illustrate how the technique is applied to data from both spacecraft. However, we emphasise that the HICAT and HIGeoCAT catalogues are compiled independently for STEREO-A and -B. The analysis applied here to generate HIGeoCAT uses only single-spacecraft geometrical-fitting techniques. An analysis of the application of analogous stereoscopic techniques (Davies et al., 2013) to CMEs observed by both HI on STEREO-A and -B will be the subject of a follow-up article.

\subsection{Catalogue Format}

The HIGeoCAT catalogue contains all of the HICAT information on each of the geometrically modelled CMEs, augmented with the kinematic parameters output from the FPF, HMF, and 


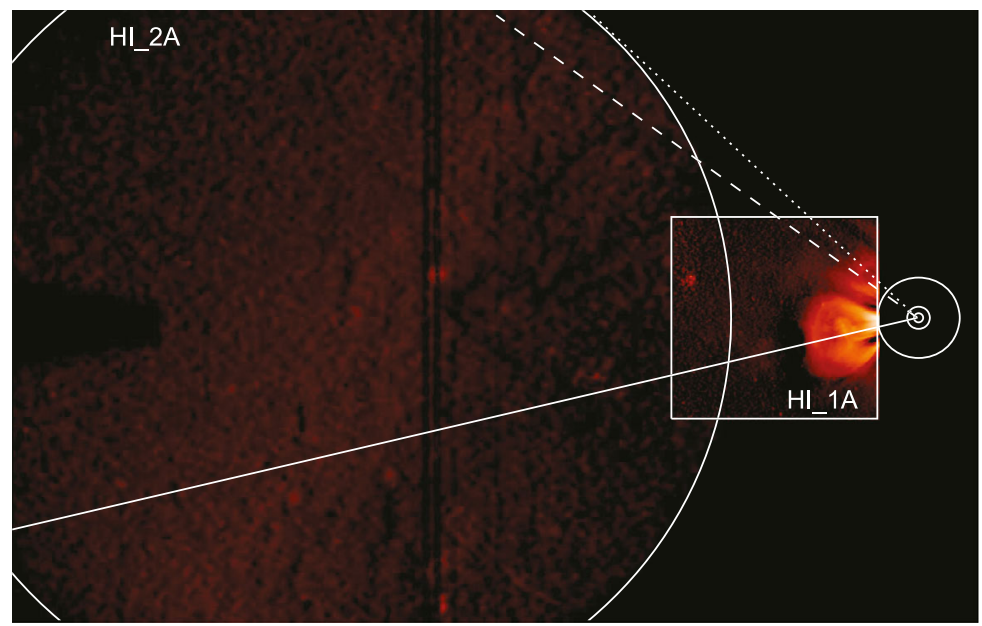

Figure 3 The combined HI-1 and HI-2 field of view at 11:29 UT on 30 January 2011. At this time, the inner HI-1A camera observes the HICAT CME HCME_A_20110130_01, and the HI-2A FOV is shown for comparison. The three concentric circles at the right of HI-1A represent the FOVs of the EUVI, COR-1, and COR-2. The solid line indicates the central PA direction of the observed CME. The dashed line represents a hypothetical CME trajectory that would cause it to be missed in the HI-2A camera, whilst the dotted line shows the path of a CME that would pass beyond the range of HI-1A before reaching the HI-2A FOV.

$\operatorname{SSEF}\left(\lambda=30^{\circ}\right)$ geometrical modelling. This information may be inspected through a display interface on the website or the full catalogue may be downloaded from the HELCATS website $^{1}$ as either a fixed-format ASCII file, a JSON file, or a VOTable XML file. The first six columns contain the HICAT information for each fitted CME:

i) a unique identifier for each $\mathrm{CME}$, constructed from a combination of some of the following parameters;

ii) the date and time (in UTC) of its first definitive observation in the HI-1 imagery (to an accuracy defined by the image cadence of 40 minutes, notwithstanding limitations in data coverage);

iii) the observing spacecraft (A or B);

iv) its northernmost PA extent (rounded to the nearest $5^{\circ}$ ), including an indicator as to whether the CME appears to extend beyond the northernmost extent of the HI-1 field of view;

v) its southernmost PA extent (rounded to the nearest $5^{\circ}$ ), including an indicator as to whether the CME appears to extend beyond the northernmost extent of the HI- 1 field of view;

vi) a measure of the confidence that the eruption is, by definition, a CME, categorised (albeit somewhat subjectively given the nature of manual cataloguing endeavours) as either good, fair, or poor.

Following this are two columns listing the PA at which the time-elongation profile fitting was performed, and the name of each file containing the raw time-elongation data, for each $\mathrm{CME}$. If there exist no data for a given $\mathrm{CME}$, because the fitting was not applied to it, these

\footnotetext{
${ }_{1}$ www.helcats-fp7.eu/catalogues/wp3_cat.html.
} 
two HIGeoCAT columns contain “-999”, as do the following columns relating to kinematic properties:

vii) the PA used in the time-elongation fitting [degrees];

viii) name of text file containing time-elongation data.

Finally, the next 24 columns contain three sets of eight best-fit parameters, for each of the FPF, SSEF, and HMF techniques, respectively. These are:

ix), xvii), xxv) CME speed [ $\left.\mathrm{km} \mathrm{s}^{-1}\right]$;

$\mathrm{x})$, xviii), xxvi) uncertainty in speed $\left[\mathrm{km} \mathrm{s}^{-1}\right]$;

$\mathrm{xi}), \mathrm{xix})$, xxvii) spacecraft-Sun-CME angle $[\phi]$ : [degrees];

xii), xx), xxviii) uncertainty in $\phi$ [degrees];

xiii), xxi), xxix) CME Heliocentric Earth Equatorial (HEEQ) Longitude [degrees];

xiv), xxii), xxx) CME HEEQ Latitude [degrees];

xv), xxiii), xxxi) CME Carrington Longitude [degrees];

xvi), xxiv), xxxii) CME launch time [UTC] from $r=0$.

Additionally, the unprocessed time-elongation data are available, in a separate text file for each CME. The CME launch time is derived from $t_{0}$ in Equation 1, which corresponds to the time at which the CME was at $r=0$, that is, the centre of the Sun. This may not seem the most intuitive choice when we could instead choose the launch time at which the leading edge was at a distance of one solar radius from the Sun centre. However, the assumption of constant CME velocity, which must be applied to arrive at this value, breaks down close to the Sun and therefore our results should not be considered an accurate representation of CME behaviour below the $4^{\circ}$ lower limit of the HI-1 FOV. Therefore, for the sake of simplicity, we chose the "launch" time to correspond to $r=0$.

\section{CME Statistical Properties}

\subsection{CME Frequency}

HICAT, as of Version 5, contains 2059 CME detections that represent all CMEs observed by the HI instruments, to the end of August 2017. The tracking of these CMEs through the HI FOVs, and the geometric modelling of their time-elongation profiles, has been applied to a subset of 1455 as part of HIGeoCAT; 801 from STEREO-A and 654 from STEREO-B. Figure 4 shows the monthly frequency of CMEs, for each spacecraft, occurring throughout the science phase of the STEREO mission as part of both HICAT and HIGeoCAT. The science phase of the mission began on 1 April 2007 and STEREO-A entered reduced operations on 18 August 2014, as it approached superior conjunction. On 27 September 2014 contact with STEREO-B was lost and, at the time of writing, no HI data have been received from it since. After 17 November 2015 full operations for STEREO-A recommenced, and we have been receiving data since this date. In Figure 4, the total height in each bin represents the number of HICAT CMEs and the darkest-shaded region represents the subset contained in HIGeoCAT. Those HICATevents that were excluded from HIGeoCAT for being considered poor (225 for STEREO-A and 204 for STEREO-B) are shown in light grey and those excluded for other reasons, as discussed in the previous section, are shown in medium grey. As was discussed in Article 1, the total number of HICAT events observed by HI-1 STEREO-A is slightly greater than that of HI-1 on STEREO-B, at least in part, due to an occasional sudden jitter experienced by the latter due to resonance between the instrument focal-plane 


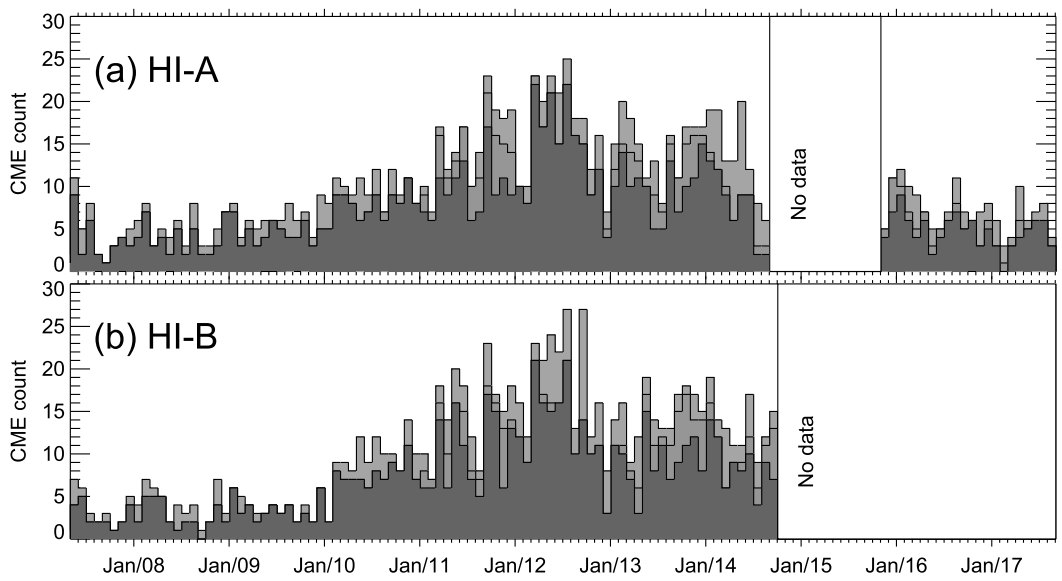

Figure 4 Monthly count of CME detections throughout the period April 2007 to August 2017 for STEREO-A (a) and STEREO-B (b). The total height of each bin represents the monthly CME count in HICAT, whilst the dark grey area shows the CMEs included in HIGeoCAT. The light grey area shows events excluded from HIGeoCAT for being considered poor and the medium grey shows events excluded due to other reasons. The data gap in panel a corresponds to the period of STEREO-A reduced operations. The data in panel $\mathbf{b}$ end when contact was lost with STEREO-B.

Figure 5 Distributions of maximum elongation to which each CME was tracked into the HI fields of view for HI on STEREO-A (a) and on STEREO-B (b).

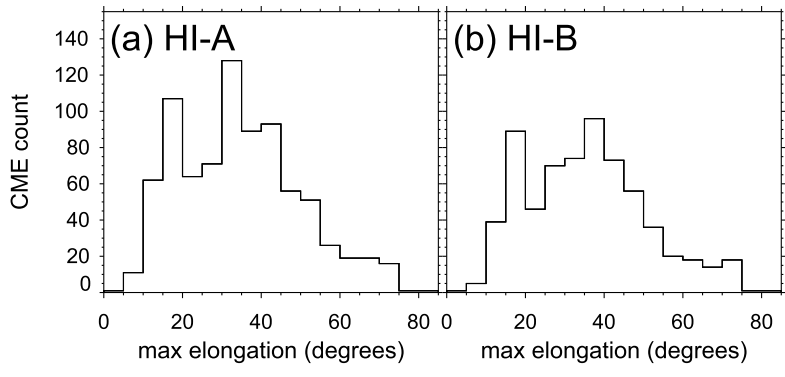

assembly and the reaction wheels. Residual background during some periods of HI- 1 data from STEREO-B is difficult to interpret, and we expect some fainter events to be missed as a result. Therefore, the number of HIGeoCAT events from the HI-B data is also generally lower than those from HI-A. The variation in the total number of HICAT events per month is consistent with established solar-cycle behaviour (Webb and Howard, 1994; St. Cyr et al., 2000, 2015), peaking at 25 per month during the first peak in sunspots of Solar Cycle 24 in 2012 and with the fewest occurring around solar minimum between Cycles 23 and 24. The total number of HIGeoCAT CMEs follows a similar trend, however, we exclude a greater proportion of events from HIGeoCAT during solar maximum. Article 1 found the ratio between good, fair and poor events to be relatively constant throughout the solar cycle; however, more high-latitude CMEs occur at solar maximum, which we are unable to track due to the gap in HI elongation coverage at PAs away from the Ecliptic.

Figure 5 shows the distributions of maximum observed elongation angles for the CMEs in HIGeoCAT using HI on STEREO-A (a) and STEREO-B (b) in five-degree bins. Both distributions show the same general features, however, they differ in that STEREO-A contains more events overall than STEREO-B. Each distribution shows a very small number of events that were tracked to less than $10^{\circ}$ elongation, and each distribution shows that no 

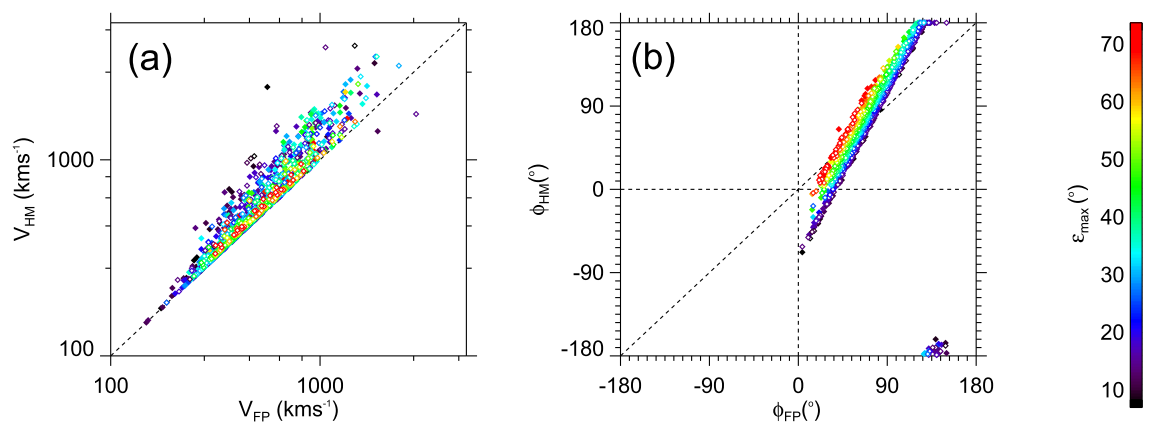

Figure 6 A comparison of the results from the FP and HM models applied to all tracked CMEs. Panel a shows a scatter-plot of $V_{\mathrm{FP}}$ versus $V_{\mathrm{HM}}$ for each HIGeoCAT CME and panel b shows $\phi_{\mathrm{FP}}$ versus $\phi_{\mathrm{HM}}$. In both cases colour represents the maximum elongation to which the CME was tracked, as indicated by the colour-bar. The filled diamonds are CMEs tracked in HI-A and open diamonds those tracked in HI-B.

events were tracked beyond $75^{\circ}$ in the HI-2 FOV. A pronounced feature in both distributions is the clear double peak. The first peak appears at $15-20^{\circ}$ for both distributions and the second at $30-35^{\circ}$ (STEREO-A) and $35-40^{\circ}$ (STEREO-B). The reason for this is that CMEs are typically easier to distinguish in HI-1 than HI-2 because the latter tend to produce noisier data. The first peaks therefore correspond to CMEs that could not be tracked beyond the edge of HI-1. Whilst the Ecliptic-extent of HI-1 is $24^{\circ}$, this limit decreases for CMEs tracked at PAs away from the Ecliptic and so the peak in fact falls below $20^{\circ}$. The secondary peaks appear low in the HI-2 FOV, where the majority of tracks that were visible in these instruments ended. Beyond this there is a decreasing tail corresponding to the events that could be tracked to greater elongation angles.

\subsection{A Comparison of Fitting Methods}

Figure 6 shows a comparison of the radial velocities and propagation angles resulting from the two extreme cases of Equation 1; FP $\left(\lambda=0^{\circ}\right)$ and $\mathrm{HM}\left(\lambda=90^{\circ}\right)$ for all HIGeoCAT detections. Panel a shows a direct comparison, for each detection, of the velocity derived from the FP and HM fitting. Panel $b$ shows a comparison between the CME apex propagation angle $[\phi]$ derived from the same methods. These plots are produced in the same format as those in Figures 3c and d of Davies et al. (2012), where a more primitive, Ecliptic-only, $\mathrm{HI}$ event list was used as a means to examine the fitting methods. Nonetheless, we find the same general results as found by Davies et al. (2012) here. In panel a of Figure 6 the majority of points lie close to the identity line, where the velocity from both methods would be equal. This effect appears more pronounced for CMEs that are tracked far into the HI FOV (those in orange/red). However, this a somewhat superficial effect because such points are far fewer in number than the shorter tracks, but have been plotted on top for emphasis. Another clear trend is that the velocity derived from FP ( $\left.V_{\mathrm{FP}}\right)$ is always less than the velocity derived from HM ( $V_{\mathrm{HM}}$ ), with just two exceptions that lie below the line $V_{\mathrm{FP}}=V_{\mathrm{HM}}$. These points correspond to a detection from HI-A (HCME_A_20120704_01) represented by the filled diamond and HI-B (HCME_B_20120606_01) represented by the open diamond. These CMEs are fitted with very few time-elongation data points, which leads us to question the veracity of the derived speeds.

Panel $b$ of Figure 6 shows data bearing a more systematic relationship between the CME propagation directions derived from $\mathrm{FP}$ and $\mathrm{HM}\left(\phi_{\mathrm{FP}}\right.$ and $\phi_{\mathrm{HM}}$, respectively) for a given 

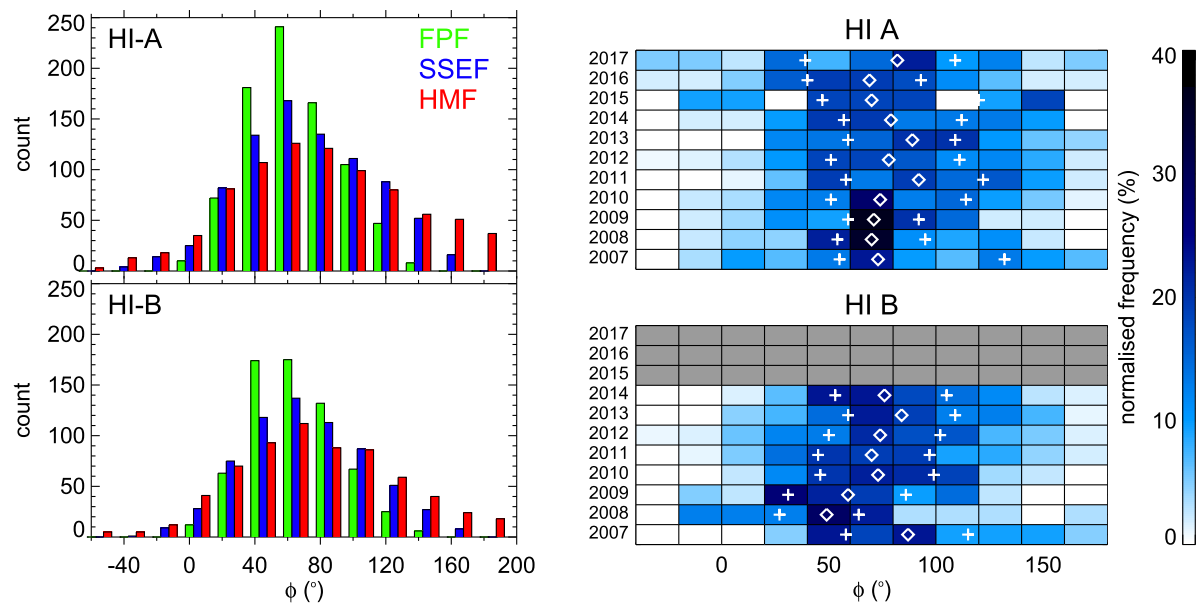

Figure 7 (Left) Distribution of observed CME propagation angle, relative to the Sun-spacecraft line, with data from HI-A (top) and HI-B (bottom), for each of the fitting methods. The green, blue, and red histograms show results using the respective FPF, SSEF, and HMF models. (Right) Stacked annual histograms of CME propagation angle, relative to the Sun-spacecraft line for STEREO-A (top) and STEREO-B (bottom) using the SSE model. Each row is normalised such that it sums to the same value (100\%). That is, it represents the percentage of the total CME in a year that fall in each $20^{\circ}$ bin. The over-plotted diamonds represent the median value and the plus symbols show the inter-quartile range.

CME. The range of $\phi_{\mathrm{HM}}$-values is broader than that of $\phi_{\mathrm{FP}}$, due to the restriction placed on the solving algorithm that the CME must impinge into the FOV of the observing instrument. Therefore $\phi_{\mathrm{FP}}$ may not fall outside the range $0-180^{\circ}$, whilst the permissible range of $\phi_{\mathrm{HM}}$ is between $\pm 180^{\circ}$. For propagation angles exceeding some $45^{\circ}, \phi_{\mathrm{HM}}$ is generally greater than $\phi_{\mathrm{FP}}$, whilst the opposite applies to angles less than $45^{\circ}$. This effect was also seen by Möstl et al. (2014), who applied FPF, SSEF, and HMF to 22 CMEs. They showed that increasing the half-width used to fit the CMEs increased, on average, the propagation angle of these CMEs away from the observing spacecraft. The colour scheme reveals a grouping of points by maximum elongation; for a given value of $\phi_{\mathrm{FP}}, \phi_{\mathrm{HM}}$ has a greater value when the CME is tracked further into the FOV. All of these features are in accordance with those observed by Davies et al. (2012), and the references therein, which contain a more thorough comparison of these fitting methods.

\subsection{CME Propagation Directions}

The left-hand panels of Figure 7 show the distributions of $\phi$, relative to the spacecraft, for HI-A (upper panel) and -B (lower panel) and for all three fitting methods (FPF: green; SSEF $\left(\lambda=30^{\circ}\right)$ : blue; HMF: red) for all HIGeoCAT CMEs. Both the HI-A and -B plots show the FP-distribution of $\phi$ to be most constrained, whilst the HM-distribution is broader. This reflects what is seen in Figure 6, which again results from the constraints placed on each of the models, and shows that the SSE-distribution lies somewhere between these extreme cases, again consistent with the results shown by Davies et al. (2012). For each of the three fitting techniques the distribution of $\phi$ peaks between $40^{\circ}$ and $80^{\circ}$ for both HI-A and HI-B. The respective median propagation direction for each of the FPF-, SSEF-, and HMF-distributions from HI-A are $78^{\circ}, 72^{\circ}$, and $84^{\circ}$. The median values from HI-B are $72^{\circ}, 69^{\circ}$, and $77^{\circ}$. This is an observational effect, where CMEs travelling close to the Thomson plateau (Tappin and 
$\mathrm{HI} \mathrm{A}$

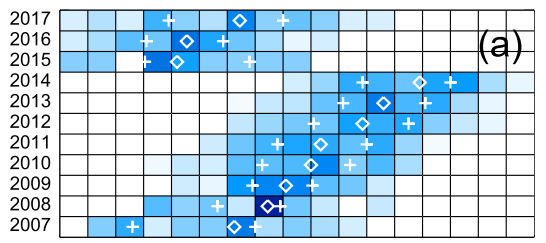

$\mathrm{HI} B$

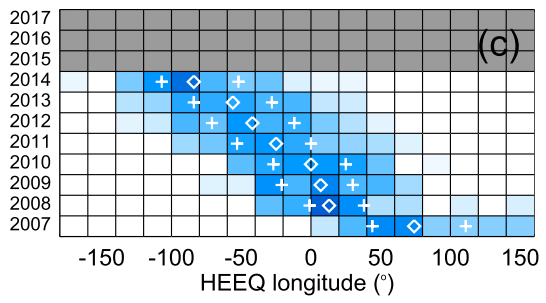

$\mathrm{HI} \mathrm{A}$

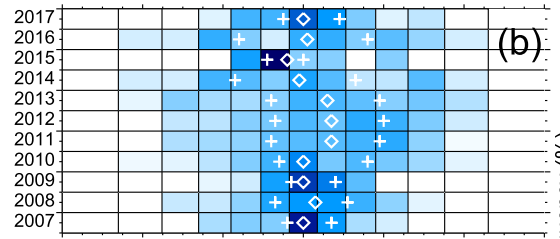

HI B

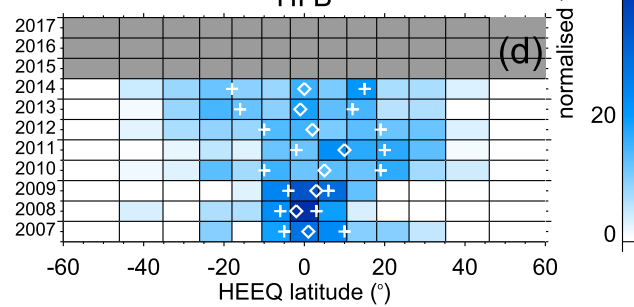

Figure 8 Stacked annual histograms of HI-A and HI-B CME HEEQ longitude, (a) and (c), and histograms of HI-A and HI-B CME HEEQ latitude, $(\mathbf{b})$ and $(\mathbf{d})$, from $\operatorname{SSE}\left(\lambda=30^{\circ}\right)$ fitting. As with Figure 7 , each row is normalised such that it sums to the same value. The areas shaded grey in (c) and (d) indicate that there are no data during these years. Median values for each annual distribution are indicated by diamonds and the first and third quartiles by pluses.

Howard, 2009) are more easily observable than those far from it. Therefore CMEs propagating at slightly less than $90^{\circ}$ from the Sun-spacecraft line are more easily observed. The right-hand panels of Figure 7 show a breakdown of the SSEF-distributions into separate years, where each has been normalised to sum to the same value, so as to remove the effect of the reduced CME rate at solar minimum. Since 2014 we have received no data from STEREO-B and therefore the following years are blank in our distribution. STEREO-A resumed full science operations towards the end of November 2015. We therefore catalogue only 16 CMEs in total during that year in HICAT (11 of which we were included in HIGeoCAT) and this is apparent in the noisier distribution we see in HI-A for 2015. There is little reason to expect that the true CME-distribution is asymmetric in HEEQ longitude, although previous studies have considered the distributions of different classes of CME in longitude. In this study we make no distinction between the CME classes, and so we may expect to see a featureless longitude distribution. The observed, albeit broad, peak is predominantly due to the enhanced visibility of CMEs travelling not too far from the Thomson surface.

Panels a and $\mathrm{c}$ of Figure 8 show the evolution of the HEEQ propagation longitude of $\mathrm{HI}-$ GeoCAT CMEs observed by HI-A and HI-B. We observe a consistent evolution of observed CME longitudes over time. As STEREO-A (panel a) drifts from the Earth in the direction of positive HEEQ longitude, so too does the longitude extent of the HI-A FOV. Consequently, the range of HEEQ longitudes over which we observe CMEs evolves also. There is an obvious discontinuity in the HI-A data between the years 2014 and 2015, which is caused by the spacecraft rotating by $180^{\circ}$ about the Sun-spacecraft line as it emerged from superior conjunction to maintain contact with Earth. Panel c shows a similar trend in HIGeoCAT CMEs observed by STEREO-B. The propagation directions of such CMEs are observed predominantly at positive HEEQ longitudes initially, but the longitude range at which they are observed becomes increasingly negative as the spacecraft progressively drifts away from the Earth. This distribution is similar to that seen in Figure 7 but with the added effect of spacecraft motion relative to the Earth. In fact we may demonstrate this cause and effect precisely; 
a linear fit to the median values in each data set (excluding the post-conjunction HI-A data) yields the relationships $\operatorname{lon}_{\mathrm{A}}=(-55 \pm 4)+(18 \pm 1) y$ and $\operatorname{lon}_{\mathrm{B}}=(53 \pm 9)-(19 \pm 2) y$, where $y$ is the number of years since 2007. These possess respective correlation coefficients of $R=0.99$ and $R=-0.97$, and we know that each spacecraft drifts by approximately $22^{\circ}$ per year, relative to Earth. The 2007 and 2014 data sets do not span an entire year, and this explains why we observe slightly smaller magnitudes of $18 \pm 1^{\circ}$ and $-19 \pm 2^{\circ}$ per year. The initial median longitudinal offset between propagation longitudes observed in HI-A $\left(-55 \pm 4^{\circ}\right)$ and HI-B $\left(53 \pm 9^{\circ}\right)$ is therefore approximately $108^{\circ}$ according to these two relationships. This is approximately equal to the spacecraft-separation angle plus the different pointing directions of the HI cameras on each spacecraft in early 2007. Likewise, we may use a similar reasoning to explain the discontinuity between the median fitted longitude in the HI-A data (panel a) between $2014\left(+78^{\circ}\right)$ and $2015\left(-96^{\circ}\right)$. Data transmission from STEREO-A was greatly reduced during the time the spacecraft moved between a HEEQ longitude of approximately $+165^{\circ}$ to $-168^{\circ}$, and the pointing direction of the cameras was rotated by $180^{\circ}$ as it exited from superior conjunction. Therefore, we would simply expect a change in sign between the observed CME longitudes before and after conjunction, which is indeed approximately what we observe.

Panels $\mathrm{b}$ and $\mathrm{d}$ of Figure 8 show the annual distributions of HEEQ latitude in a similar format to that in which the longitudes are displayed. However, the bin-widths are scaled by $1 / \cos$ (latitude), such that they each correspond to an approximately equal area on the solar surface. For each year of both HI-A and HI-B observations, we see that the median latitude of CME propagation is very close to the $0^{\circ}$. In fact, the median latitude for all HI-A and HI-B CMEs is $2^{\circ}$ and $3^{\circ}$, respectively. There is no significant difference between the distributions for each spacecraft during the period when both were transmitting data. For both spacecraft, a well-established annual trend presents itself; CMEs near solar minimum (2009) appear confined around the Equator, whilst the distribution becomes broader at the solar maximum of Cycle 24 (2012 - 2014). Again note that the HI-A 2015 data are particularly noisy because they only contain 11 CMEs. Subsequently, we see the distribution narrow between 2016 and 2017 as we again approach solar minimum. This is consistent with solar-cycle behaviour established in many previous studies, for example Burkepile and St. Cyr (1993), St. Cyr et al. (2000), and Yashiro et al. (2004).

\subsection{CME Speeds}

Figure 9 shows the distribution of CME speeds from each of the fitting methods for all HIGeoCAT CMEs. As with the distribution of angles in Figure 7, we see a trend that the FPF speeds (green) are constrained, whilst the HMF (red) distribution is broader. The peak speeds all lie in the $300-350 \mathrm{~km} \mathrm{~s}^{-1}$ and $350-400 \mathrm{~km} \mathrm{~s}^{-1}$ bins. Again, this reflects the behaviour seen in Figure 6, where a given CME is faster when modelled with HMF rather than FPF. In addition, the SSEF-distribution appears again to be intermediate with respect to its peak and breadth with regards to the FP and $\mathrm{HM}$ distributions. The median speeds that we observe are $v_{\mathrm{A}}=443$ and $v_{\mathrm{B}}=460 \mathrm{~km} \mathrm{~s}^{-1}$ for the FPF, $v_{\mathrm{A}}=474$ and $v_{\mathrm{B}}=481 \mathrm{~km} \mathrm{~s}^{-1}$ for $\operatorname{SSEF}\left(\lambda=30^{\circ}\right)$, and $v_{\mathrm{A}}=495$ and $v_{\mathrm{B}}=504 \mathrm{~km} \mathrm{~s}^{-1}$ for HMF. This is quite consistent with established CME speeds determined from coronagraphs; Yurchyshyn et al. (2005), for example, show a distribution of CDAW CME speeds that peaks at $350 \mathrm{~km} \mathrm{~s}^{-1}$ with a long tail extending to CMEs exceeding $1000 \mathrm{~km} \mathrm{~s}^{-1}$ during the ascending phase of Solar Cycle 23. Yashiro et al. (2004) study CMEs from the same period as Yurchyshyn et al. (2005) using the LASCO CDAW CME list and find that average CME speeds range from $281 \mathrm{~km} \mathrm{~s}^{-1}$ at 


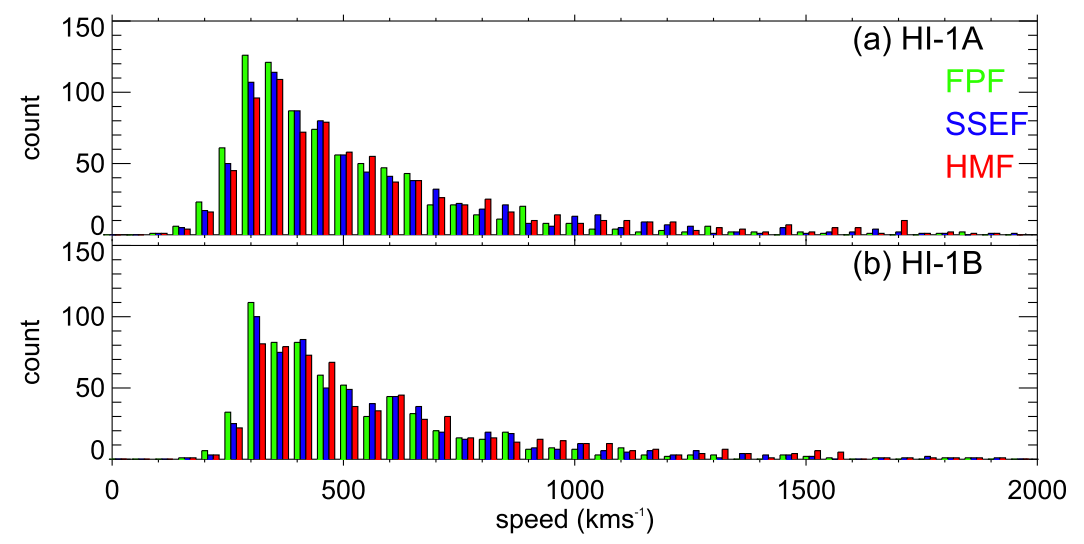

Figure 9 Distributions of best-fit radial speeds from HI-A (a) and HI-B (b) for FPF ( green), $\operatorname{SSEF}\left(\lambda=30^{\circ}\right.$, blue) and HMF (red).

solar minimum (1996) to $502 \mathrm{~km} \mathrm{~s}^{-1}$ at maximum (2001). As a means to study the solarcycle variation in speeds within our dataset we make a direct comparison of the annual distributions from CDAW, COR-2, and HIGeoCAT.

A stacked annual histogram of the annual distribution of CME speeds from SSEF are shown in Figure 10, in addition to the corresponding values from the COR-2 catalogue (Vourlidas et al., 2017: solar.jhuapl.edu/Data-Products/COR-CME-Catalog.php) and LASCO CDAW catalogue (Yashiro et al., 2004: cdaw.gsfc.nasa.gov/CME_list/) for the same period. It should be emphasised that, as with the previous figures, each annual distribution has been normalised to a total of $100 \%$. The CDAW catalogue contains speeds produced using a linear fit to the CME height-time profile, assuming it is observed in the sky plane. In addition to this, they provide the speed at $20 \mathrm{R}_{\odot}$ determined using a second-order fit. Here we have used the former, which we believe to be more consistent with our catalogue. Similarly, Vourlidas et al. (2017) produce both a linear-fit velocity and second-order fit velocity at $10 \mathrm{R}_{\odot}$. However, only the second-order fit is contained in their COR-2 catalogue and so these are the data that we have used in Figure 10. From the LASCO catalogue we have excluded poor events and events with a PA width less than $20^{\circ}$, in our analysis. Neither have we included the COR-2 events where the PA width is less than $20^{\circ}$. The number of resulting CMEs is significantly greater in the CDAW catalogue than in both HIGeoCAT and the COR-2 catalogues; an effect that is masked by normalising the data. The apparent smoothness of the annual CDAW speed distributions, compared to those of HI and COR, is thus the result of this difference in number of events. A distinct contrast between the CDAW-distribution and both those of $\mathrm{HI}$ is the difference in the number of slow events. There are very few CMEs with speeds less than $200 \mathrm{~km} \mathrm{~s}^{-1}$ seen in the HI data throughout the duration of the STEREO mission. Conversely the CDAW catalogue consistently shows CMEs slower than this during the same period. During solar-maximum years, these slow speeds generally represent the tail-end of the CDAW-distributions; however, during a solar minimum they comprise to a significant fraction of the total observed. These slower speeds observed in CDAW compared to HIGeoCAT are believed to be a result of the different methods used to track CMEs in the two catalogues; the sky-plane assumption applied to the former (Gopalswamy et al., 2009) causes the derived speeds to be an underestimate of the true speed. Both COR-2A and COR-2B show the lowest annual speed distributions during solar minimum compared to the HI-A-, HI-B-, or LASCO-distributions. At solar maximum, 
HI A

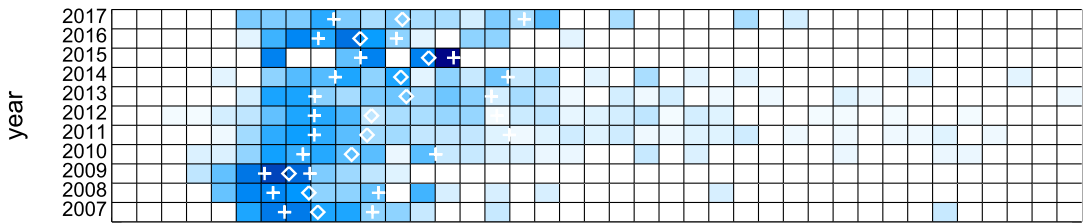

HI B

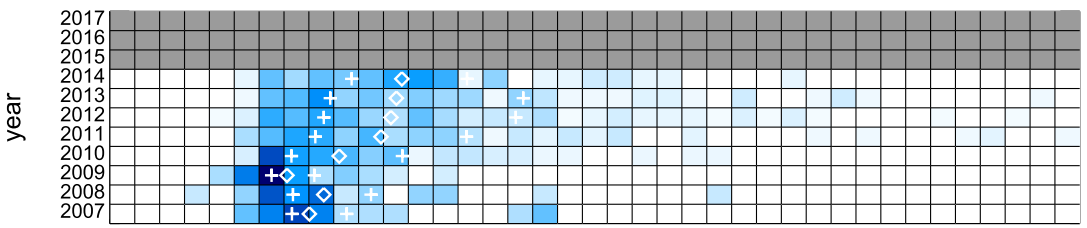

COR 2A

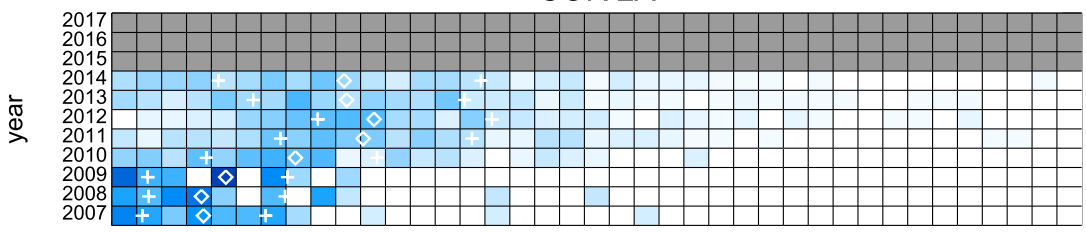

COR 2B

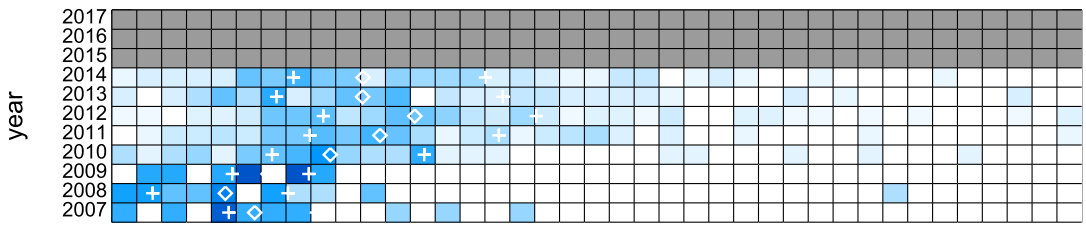

\section{LASCO}

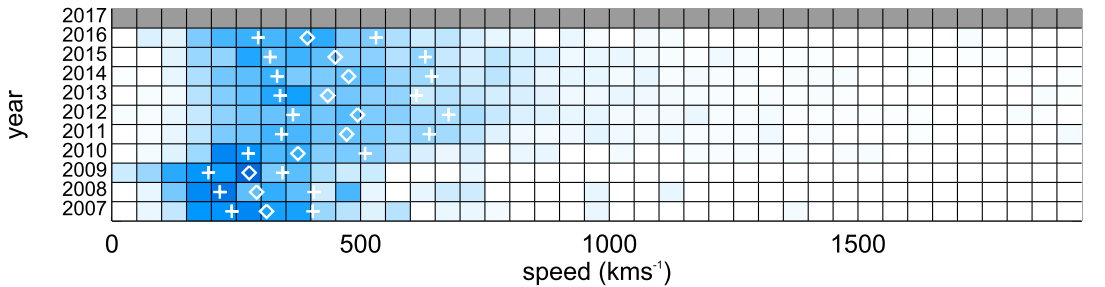

Figure 10 Stacked annual histograms of fitted CME speeds. The STEREO-A and -B data are produced using all events from the HIGeoCAT, fitted using the SSEF. LASCO data from the Vourlidas et al. (2017) COR-2 catalogue during the same period are shown for comparison, as are those from the LASCO CDAW catalogue. Each plot has been normalised such that each annual distribution sums to the same value.

the mean speeds from the COR-distributions are generally greater than those from LASCO, but less than those from HI. As for the CDAW catalogue, the COR-2 catalogue uses the sky-plane assumption to derive CME speeds, which accounts for why the COR-2 speeds are typically lower than those derived from the HI data. The COR-2 values represent the speed at $10 \mathrm{R}_{\odot}$ using a second-order fit, whilst those in the LASCO catalogue are derived from a linear fit. In the COR-2A and COR-2B catalogue we find that the respective numbers of events with a positive acceleration are $44 \%$ and $42 \%$ and the respective median acceleration values are 0 and $-2 \mathrm{~ms}^{-2}$. Therefore we would not expect these COR-2 speeds to be 

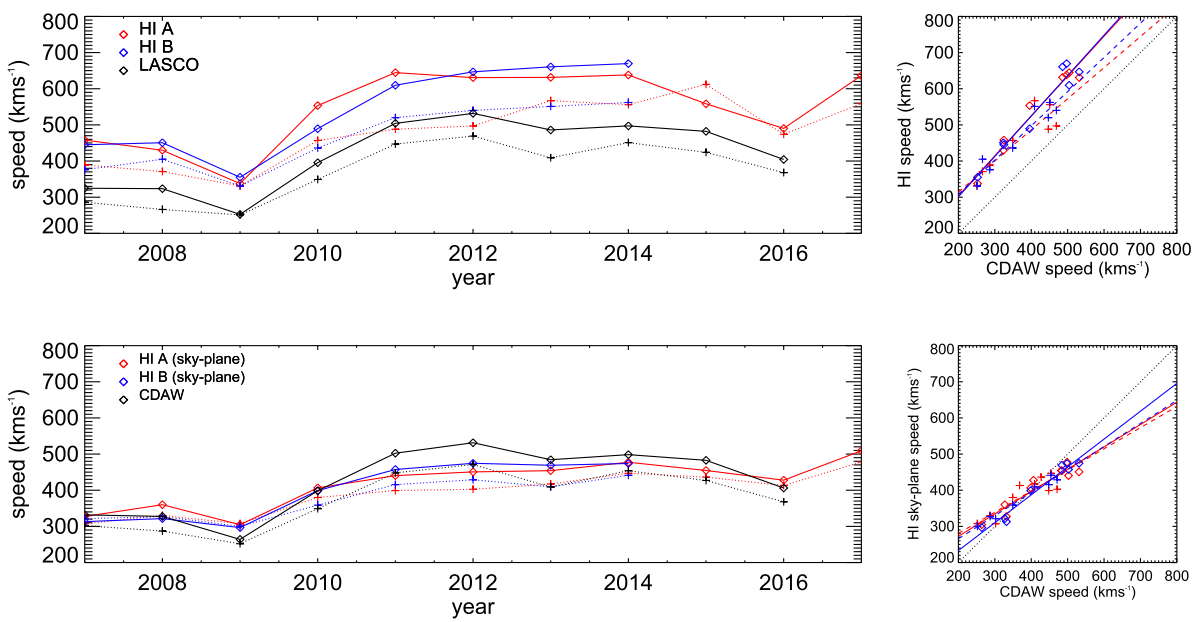

Figure 11 (Top left) Annual average radial CME speeds from the HIGeoCAT catalogue for HI-A (red) and HI-B (blue). The CDAW values are shown in black. Mean speeds are indicated by diamonds connected by a solid line and median speeds by crosses connected by a dashed line. (Top right) A direct comparison between the mean annual speed of CMEs in the CDAW catalogue and the corresponding mean annual speeds of HIGeoCAT CMEs. Again HI-A and -B are represented, respectively, by red and blue. The mean (median) annual speeds are defined by diamonds (crosses) and solid (dashed) regression lines. (Bottom left and bottom right) The same format as the panels above, however, the HI speeds have been determined assuming that observed CMEs are moving in the sky plane.

greater if they were taken at higher altitudes, such as those observed by HI. Vourlidas et al. (2017) compare their COR-2 speeds to those derived in CDAW, however, they only consider yearly averages, which they conclude are consistent between both catalogues, and they do not study annual distributions such as those in Figure 10. There is a general trend seen in all of the five sets of the HI-A, HI-B, COR-2A, COR-2B, and LASCO data for both the median speed and the range to be greater at solar maximum and smallest at solar minimum, which is again consistent with established CME behaviour. These annual trends for the HIGeoCAT and CDAW catalogues are compared directly in Figure 11.

The top left-hand panel of Figure 11 shows the annual mean and median speeds from each of the HI-A, HI-B, and CDAW catalogues from 2007 to 2014. The difference in speeds between those derived from HI and those from LASCO is again clear; the mean and median speeds from both HI spacecraft are consistently greater than those of the CDAW catalogue. All three of HI-A, HI-B and LASCO exhibit a minimum in 2009 with an increase thereafter, correlating with the variation in sunspot number of Solar Cycle 24. The top right-hand scatter-plot shows a direct comparison of both the annual mean and median values from HI-A and LASCO in red and a comparison of the annual mean and median values from HI-B and LASCO in blue. The equations for each of the regression lines are shown explicitly in Table 1 . There is a clear systematic difference where the HI values are greater than those from LASCO. This is again attributed to the sky-plane effect; the difference in speeds of CMEs in the CDAW and HIGeoCAT catalogues is predominantly due to the sky-plane assumption in the former. We recalculate the HIGeoCAT speeds using the same sky-plane assumption that is used to produce the CDAW catalogue. These results are presented in the bottom panels of Figure 11.

The bottom two panels of Figure 11 shows the result of taking the same time-elongation data for each CME used in HIGeoCAT and assuming they are represented by a point source 
Table 1 Tabulated results from the lines fitted to the right-hand scatter-plot in Figure 11. The notation $\bar{v}$ and $\tilde{v}$ represents the respective mean and median velocities and the subscripts $A$, $B$, and $C$ correspond to HI-A, HI-B, and CDAW.

\begin{tabular}{lll}
\hline Instrument & HIGeoCAT Speed $\left[\mathrm{km} \mathrm{s}^{-1}\right]$ & $R$-value \\
\hline HI-A & $\bar{v}_{A}=(1.1 \pm 0.1) \bar{v}_{C}+(68 \pm 51)$ & 0.96 \\
& $\tilde{v}_{A}=(1.0 \pm 0.2) \tilde{v}_{C}+(99 \pm 84)$ & 0.85 \\
HI-B & $\bar{v}_{B}=(1.2 \pm 0.1) \bar{v}_{C}+(55 \pm 49)$ & 0.97 \\
& $\tilde{v}_{B}=(1.0 \pm 0.1) \tilde{v}_{C}+(93 \pm 48)$ & 0.96 \\
& Sky-plane Speed $\left[\mathrm{km} \mathrm{s}^{-1}\right]$ & \\
HI-A & $\bar{v}_{A}=(0.6 \pm 0.1) \bar{v}_{C}+(149 \pm 32)$ & 0.95 \\
& $\tilde{v}_{A}=(0.6 \pm 0.1) \tilde{v}_{C}+(164 \pm 43)$ & 0.88 \\
HI-B & $\bar{v}_{B}=(0.8 \pm 0.1) \bar{v}_{C}+(80 \pm 26)$ & 0.98 \\
& $\tilde{v}_{B}=(0.6 \pm 0.1) \tilde{v}_{C}+(138 \pm 17)$ & 0.99 \\
\hline
\end{tabular}

observed in the sky plane. That is, we determine CME speed using the same method as in the CDAW catalogue. For a given CME we produce a height-time profile and apply a linear fit to derive a CME speed. This is equivalent to running FPF with $\phi=90^{\circ}$. The result is that the yearly average HIGeoCAT speeds, both mean and median, match the CDAW results much more closely; however, some differences remain. The scatter-plot on the bottom right of Figure 11 illustrates how much closer the values are compared to Figure 11. During solar-minimum years, when CMEs are slower in general, CDAW speeds tend to be slightly slower than HIGeoCAT speeds; during solar-maximum years, when CMEs tend to be faster, the inverse is true. This is reflected in the gradients of the regression lines, the equations of which are again listed explicitly in Table 1 . This may be explained by CME acceleration within the LASCO FOV, and the lack of it within the HI FOV. Yashiro et al. (2004) show that the very slowest CDAW CMEs are typically accelerating towards the ambient solar-wind speed, whilst the fastest are decelerating toward it. Therefore we see a flatter distribution of average CME speeds over the solar cycle in HIGeoCAT, where much of this acceleration has already occurred.

\section{Summary}

2059 CMEs were identified in Version 5 of HICAT, of which a subset of 1455 have had single-spacecraft geometrical fitting applied to determine their kinematic properties. In Article 1 (Harrison et al., 2018), which presents the generation of the HICAT catalogue and a statistical analysis of its events, CME frequency was found to correlate with SSN over Solar Cycle 24 and the number of events observed by STEREO-B was found to be marginally less than observed by STEREO-A due to HI-1B jitter. The fraction of "poor" events, which are excluded from the kinematic modelling in creating HIGeoCAT, do not differ significantly between HI-1A and HI-1B. As such, we find similar trends in the distribution of tracked events as part of this study. We observe an increase toward solar maximum and slightly fewer events overall that are tracked for STEREO-B. A further factor that reduced the number of events that we were able to track at solar maximum is that CME launch sites tend to cover a greater latitudinal range during that phase of the cycle. As a consequence, many CMEs propagate at PAs far from the Ecliptic and cannot be tracked to a sufficiently large elongation from the Sun and are hence excluded from further analysis.

A comparison between the results from the three single-spacecraft fitting methods applied to derive the kinematic properties (FPF, SSEF $\left(\lambda=30^{\circ}\right)$, and HMF) is carried out on 
the resulting CME propagation directions and speeds. We find that the median propagation directions lie between $69^{\circ}$ and $84^{\circ}$ from the Sun-spacecraft line. This tendency to observe CMEs at angles slightly less than $90^{\circ}$ is because they are more likely to lie closer to the Thomson plateau. The HMF-distribution is broadest, whilst the FPF-distribution is most constrained. The annual distributions of CME propagation angle derived from SSEF show no significant variation over the solar cycle. When the CME propagation angle $[\phi]$ (that is, measured relative to the Sun-spacecraft line) is converted to HEEQ longitude, the distributions show little variation between years, or between spacecraft, except that they drift by $22^{\circ}$ and in opposite directions for CMEs observed by HI-A and HI-B. This is of course consistent with the motion of the spacecraft relative to Earth. Between 2014 and 2015 there is a large change in observed HEEQ longitude due to the fact that the spacecraft was rotated through $180^{\circ}$ to maintain communications with Earth. The distributions of HEEQ latitudes, when plotted annually show a trend consistent with the established solar-cycle variation; at solar minimum they are sharply peaked around the Equator, whilst at maximum they show a much broader distribution. The latitude range of these distributions at solar maximum is likely truncated due to the limited PA range of the HI instruments and the fact that more CMEs originating at higher latitudes are omitted from the catalogue.

Annual CME speed distributions from HIGeoCAT, using the SSEF technique, are compared to CDAW speeds from the same period. The HI-distributions show consistently higher speeds, but with otherwise similar features; a sharp peak at the low end of the distribution and a long tail of higher-speed CMEs. The speeds from the Vourlidas et al. (2017) COR-2 catalogue are also compared to HIGeoCAT and CDAW. These are found to be much lower, which is thought to be due to a different method used to determine speeds in the COR-2 catalogue. CDAW and HIGeoCAT use a linear fit to time-height or time-elongation profiles, assuming a constant speed. The COR- 2 catalogue provides a $10 \mathrm{R}_{\odot}$ speed derived from a second-order fit to the time-height data. The solar-cycle variation seen in HI speed distributions is consistent with CDAW, COR-2, and with established CME behaviour. We see a much narrower speed distribution at solar minimum, particularly 2009, and lower values overall in all catalogues. At solar maximum the median and range of speeds is greater. When compared directly, the mean speed each year in HI-A (B) is found to be $\approx 62(117) \mathrm{km} \mathrm{s}^{-1}$ greater than CDAW, whilst the median speeds are $\approx 122(161) \mathrm{km} \mathrm{s}^{-1}$ greater. This is due to the use of the sky-plane approximation, which is known to underestimate speeds when a CME is travelling out of the sky plane. When the sky-plane approximation is also applied to the analysis of HI data, the speed distributions between HI and CDAW show a much better agreement, demonstrating that invoking the sky-plane approximation is responsible for much of the difference between CDAW and HIGeoCAT speeds. However, there is still a small difference even once this effect has been taken into account. During solar-minimum years, CDAW speeds tend to lower values than those in HIGeoCAT and during solar-maximum years the inverse is true. That is, the HIGeoCAT speed distribution is flatter over the solar cycle than those in CDAW. This is because CMEs experience more acceleration in the LASCO FOV, typically towards the ambient solar-wind speed. Therefore we would expect less variation in speeds by the time CMEs reach the HI FOV. However, some CMEs are known to continue to accelerate beyond the FOV of LASCO. This is a subject that is to be dealt with in a follow-up, where stereoscopic methods are applied to a further subset of CMEs that are observable by both HI-A and HI-B, allowing acceleration estimates to be made.

Acknowledgements We acknowledge support from the European Union FP7-SPACE-2013-1 programme for the HELCATS project (\#606692). The HI instruments on STEREO were developed by a consortium that comprised the Rutherford Appleton Laboratory (UK), the University of Birmingham (UK), Centre Spatial de Liège (CSL, Belgium) and the Naval Research Laboratory (NRL, USA). The STEREO/SECCHI project, 
of which HI is a part, is an international consortium led by NRL. We recognise the support of the UK Space Agency for funding STEREO/HI operations in the UK. We also acknowledge use of the CDAW SOHO/LASCO event catalogue (cdaw.gsfc.nasa.gov/CME_list/), which is generated and maintained at the CDAW Data Centre, by NASA and the Catholic University of America in cooperation with NRL, and the STEREO/SECCHI/COR2 CME catalogue (solar.jhuapl.edu/Data-Products/COR-CME-Catalog.php) that is generated and maintained by John Hopkins University Applied Physics Laboratory, in collaboration with NRL and the NASA Goddard Space Flight Centre. C. Möstl also thanks the Austrian Science Fund (FWF): [P26174-N27].

Disclosure of Potential Conflicts of Interest The authors declare that they have no conflicts of interest.

Publisher's Note Springer Nature remains neutral with regard to jurisdictional claims in published maps and institutional affiliations.

Open Access This article is distributed under the terms of the Creative Commons Attribution 4.0 International License (http://creativecommons.org/licenses/by/4.0/), which permits unrestricted use, distribution, and reproduction in any medium, provided you give appropriate credit to the original author(s) and the source, provide a link to the Creative Commons license, and indicate if changes were made.

\section{References}

Aschwanden, M.J., Wülser, J.-P., Nitta, N.V., Lemen, J.R., Freeland, S., Thompson, W.T.: 2014, STEREO/Extreme Ultraviolet Imager (EUVI) event catalog 2006-2012. Solar Phys. 289, 919. DOI.

Barnard, L., Scott, C., Owens, M., Lockwood, M., Tucker-Hood, K., Thomas, S., Crothers, S., Davies, J.A., Harrison, R., Lintott, C., Simpson, R., O’Donnell, J., Smith, A.M., Waterson, N., Bamford, S., Romeo, F., Kukula, M., Owens, B., Savani, N., Wilkinson, J., Baeten, E., Poeffel, L., Harder, B.: 2014, The Solar Stormwatch CME catalogue: Results from the first space weather citizen science project. Space Weather 12(12), 657. DOI.

Brueckner, G.E., Howard, R.A., Koomen, M.J., Korendyke, C.M., Michels, D.J., Moses, J.D., Socker, D.G., Dere, K.P., Lamy, P.L., Llebaria, A., Bout, M.V., Schwenn, R., Simnett, G.M., Bedford, D.K., Eyles, C.J.: 1995, The Large Angle Spectroscopic Coronagraph (LASCO). Solar Phys. 162, 357. DOI. ADS.

Burkepile, J.T., St. Cyr, O.C.: 1993, A revised and expanded catalogue of mass ejections observed by the Solar Maximum Mission coronagraph. NASA STI/Recon Technical Report N 93. ADS.

Byrne, J.P., Maloney, S.A., McAteer, R.T.J., Refojo, J.M., Gallagher, P.T.: 2010, Propagation of an Earthdirected coronal mass ejection in three dimensions. Nat. Commun. 1, 74. DOI. ADS.

Byrne, J.P., Morgan, H., Habbal, S.R., Gallagher, P.T.: 2012, Automatic detection and tracking of coronal mass ejections. II. Multiscale filtering of coronagraph images. Astrophys. J. 752, 145. DOI. ADS.

Davies, J.A., Harrison, R.A., Perry, C.H., Möstl, C., Lugaz, N., Rollett, T., Davis, C.J., Crothers, S.R., Temmer, M., Eyles, C.J., Savani, N.P.: 2012, A self-similar expansion model for use in solar wind transient propagation studies. Astrophys. J. 750, 23. DOI. ADS.

Davies, J.A., Perry, C.H., Trines, R.M.G.M., Harrison, R.A., Lugaz, N., Möstl, C., Liu, Y.D., Steed, K.: 2013, Establishing a stereoscopic technique for determining the kinematic properties of solar wind transients based on a generalized self-similarly expanding circular geometry. Astrophys. J. 777, 167. DOI. ADS.

Davis, C.J., Kennedy, J., Davies, J.A.: 2010, Assessing the accuracy of CME speed and trajectory estimates from STEREO observations through a comparison of independent methods. Solar Phys. 263, 209. DOI. ADS.

Elmore, D.F., Burkepile, J.T., Darnell, J.A., Lecinski, A.R., Stanger, A.L.: 2003, Calibration of a groundbased solar coronal polarimeter. In: Fineschi, S. (ed.) Polarimetry in Astronomy 4843, 66. DOI. ADS.

Eyles, C.J., Simnett, G.M., Cooke, M.P., Jackson, B.V., Buffington, A., Hick, P.P., Waltham, N.R., King, J.M., Anderson, P.A., Holladay, P.E.: 2003, The Solar Mass Ejection Imager (SMEI). Solar Phys. 217, 319. DOI. ADS.

Eyles, C.J., Harrison, R.A., Davis, C.J., Waltham, N.R., Shaughnessy, B.M., Mapson-Menard, H.C.A., Bewsher, D., Crothers, S.R., Davies, J.A., Simnett, G.M., Howard, R.A., Moses, J.D., Newmark, J.S., Socker, D.G., Halain, J.-P., Defise, J.-M., Mazy, E., Rochus, P.: 2009, The heliospheric imagers onboard the STEREO mission. Solar Phys. 254, 387. DOI. ADS.

Fisher, R.R., Lee, R.H., MacQueen, R.M., Poland, A.I.: 1981, New Mauna Loa coronagraph systems. Appl. Opt. 20, 1094. DOI. ADS. 
Gopalswamy, N., Yashiro, S., Michalek, G., Stenborg, G., Vourlidas, A., Freeland, S., Howard, R.: 2009, The SOHO/LASCO CME catalog. Earth Moon Planets 104(1), 295.

Gopalswamy, N., Akiyama, S., Yashiro, S., Xie, H., Mäkelä, P., Michalek, G.: 2014a, Anomalous expansion of coronal mass ejections during Solar Cycle 24 and its space weather implications. Geophys. Res. Lett. 41, 2673. DOI. ADS.

Gopalswamy, N., Xie, H., Akiyama, S., Mäkelä, P.A., Yashiro, S.: 2014b, Major solar eruptions and highenergy particle events during Solar Cycle 24. Earth Planets Space 66, 104. DOI. ADS.

Gopalswamy, N., Xie, H., Akiyama, S., Mäkelä, P., Yashiro, S., Michalek, G.: 2015, The peculiar behavior of halo coronal mass ejections in Solar Cycle 24. Astrophys. J. 804, L23. DOI. ADS.

Gosling, J.T., Hildner, E., MacQueen, R.M., Munro, R.H., Poland, A.I., Ross, C.L.: 1974, Mass ejections from the Sun - A view from SKYLAB. J. Geophys. Res. 79, 4581. DOI. ADS.

Gosling, J.T., McComas, D.J., Phillips, J.L., Bame, S.J.: 1991, Geomagnetic activity associated with Earth passage of interplanetary shock disturbances and coronal mass ejections. J. Geophys. Res. 96(A5), 7831. DOI.

Harrison, R.A., Davies, J.A., Möstl, C., Liu, Y., Temmer, M., Bisi, M.M., Eastwood, J.P., de Koning, C.A., Nitta, N., Rollett, T., Farrugia, C.J., Forsyth, R.J., Jackson, B.V., Jensen, E.A., Kilpua, E.K.J., Odstrcil, D., Webb, D.F.: 2012, An analysis of the origin and propagation of the multiple coronal mass ejections of 2010 August 1. Astrophys. J. 750, 45. DOI. ADS.

Harrison, R.A., Davies, J.A., Barnes, D., Byrne, J.P., Perry, C.H., Bothmer, V., Eastwood, J.P., Gallagher, P.T., Kilpua, E.K.J., Möstl, C., Rodriguez, L., Rouillard, A.P., Odstrčil, D.: 2018, CMEs in the heliosphere: I. A statistical analysis of the observational properties of CMEs detected in the heliosphere from 2007 to 2017 by STEREO/HI-1. Solar Phys. 293, 77. DOI. ADS.

Howard, R.A., Sheeley, N.R. Jr., Michels, D.J., Koomen, M.J.: 1985, Coronal mass ejections: 1979-1981. J. Geophys. Res. 90, 8173. DOI. ADS.

Howard, R.A., Moses, J.D., Vourlidas, A., Newmark, J.S., Socker, D.G., Plunkett, S.P., Korendyke, C.M., Cook, J.W., Hurley, A., Davila, J.M., Thompson, W.T., St. Cyr, O.C., Mentzell, E., Mehalick, K., Lemen, J.R., Wuelser, J.P., Duncan, D.W., Tarbell, T.D., Wolfson, C.J., Moore, A., Harrison, R.A., Waltham, N.R., Lang, J., Davis, C.J., Eyles, C.J., Mapson-Menard, H., Simnett, G.M., Halain, J.P., Defise, J.M., Mazy, E., Rochus, P., Mercier, R., Ravet, M.F., Delmotte, F., Auchere, F., Delaboudiniere, J.P., Bothmer, V., Deutsch, W., Wang, D., Rich, N., Cooper, S., Stephens, V., Maahs, G., Baugh, R., McMullin, D., Carter, T.: 2008, Sun Earth Connection Coronal and Heliospheric Investigation (SECCHI). Space Sci. Rev. 136, 67. DOI. ADS.

Hundhausen, A.J., Sawyer, C.B., House, L., Illing, R.M.E., Wagner, W.J.: 1984, Coronal mass ejections observed during the solar maximum mission - Latitude distribution and rate of occurrence. J. Geophys. Res. 89, 2639. DOI. ADS.

Kahler, S.W., Webb, D.F.: 2007, V arc interplanetary coronal mass ejections observed with the Solar Mass Ejection Imager. J. Geophys. Res. 112, A09103. DOI. ADS.

Kilpua, E.K.J., Schwenn, R., Bothmer, V., Koskinen, H.E.J.: 2005, Properties and geoeffectiveness of magnetic clouds in the rising, maximum and early declining phases of Solar Cycle 23. Ann. Geophys. 23, 4491.

Kilpua, E.K.J., Balogh, A., von Steiger, R., Liu, Y.D.: 2017, Geoeffective properties of solar transients and stream interaction regions. Space Sci. Rev. 212(3), 1271. DOI.

Kirnosov, V., Chang, L.-C., Pulkkinen, A.: 2016, Combining STEREO SECCHI COR2 and HI1 images for automatic CME front edge tracking. J. Space Weather Space Clim. 6(27), A41. DOI. ADS.

Lugaz, N.: 2010, Accuracy and limitations of fitting and stereoscopic methods to determine the direction of coronal mass ejections from Heliospheric Imagers observations. Solar Phys. 267, 411. DOI. ADS.

Lugaz, N., Vourlidas, A., Roussev, I.I.: 2009, Deriving the radial distances of wide coronal mass ejections from elongation measurements in the heliosphere - application to CME-CME interaction. Ann. Geophys. 27, 3479. DOI. ADS.

MacQueen, R.M., Csoeke-Poeckh, A., Hildner, E., House, L., Reynolds, R., Stanger, A., Tepoel, H., Wagner, W.: 1980, The High Altitude Observatory Coronagraph/Polarimeter on the Solar Maximum Mission. Solar Phys. 65, 91. DOI. ADS.

Markwardt, C.B.: 2009, Non-linear least-squares fitting in IDL with MPFIT. In: Bohlender, D.A., Durand, D., Dowler, P. (eds.) Astronomical Data Analysis Software and Systems XVIII CS-411, Astron. Soc. Pacific, San Francisco, 251. ADS.

Minnaert, M.: 1930, On the continuous spectrum of the corona and its polarisation. With 3 figures. Z. Astrophys. 1, 209. ADS.

Moré, J.J.: 1978, The Levenberg-Marquardt algorithm: Implementation and theory. In: Numerical Analysis. Lect. Notes Math. 630, 105. 3-540-08538-6 (print), 3-540-35972-9 (e-book). DOI. 
Morgan, H., Byrne, J.P., Habbal, S.R.: 2012, Automatically detecting and tracking coronal mass ejections. I. Separation of dynamic and quiescent components in coronagraph images. Astrophys. J. 752, 144. DOI. ADS.

Möstl, C., Davies, J.A.: 2013, Speeds and arrival times of solar transients approximated by self-similar expanding circular fronts. Solar Phys. 285, 411. DOI. ADS.

Möstl, C., Temmer, M., Rollett, T., Farrugia, C.J., Liu, Y., Veronig, A.M., Leitner, M., Galvin, A.B., Biernat, H.K.: 2010, STEREO and Wind observations of a fast ICME flank triggering a prolonged geomagnetic storm on 5-7 April 2010. Geophys. Res. Lett. 37, L24103. DOI. ADS.

Möstl, C., Amla, K., Hall, J.R., Liewer, P.C., Jong, E.M.D., Colaninno, R.C., Veronig, A.M., Rollett, T., Temmer, M., Peinhart, V., Davies, J.A., Lugaz, N., Liu, Y.D., Farrugia, C.J., Luhmann, J.G., Vršnak, B., Harrison, R.A., Galvin, A.B.: 2014, Connecting speeds, directions and arrival times of 22 coronal mass ejections from the Sun to 1 AU. Astrophys. J. 787(2), 119. DOI.

Nelder, J.A., Mead, R.: 1965, A simplex method for function minimization. Comput. J. 7(4), 308. DOI.

Olmedo, O., Zhang, J., Wechsler, H., Poland, A., Borne, K.: 2008, Automatic detection and tracking of coronal mass ejections in coronagraph time series. Solar Phys. 248, 485. DOI. ADS.

Pant, V., Willems, S., Rodriguez, L., Mierla, M., Banerjee, D., Davies, J.A.: 2016, Automated detection of coronal mass ejections in STEREO heliospheric imager data. Astrophys. J. 833(1), 80.

Richardson, I., Cane, H.: 2012, Near-Earth solar wind flows and related geomagnetic activity during more than four solar cycles (1963-2011). J. Space Weather Space Clim. 2.

Robbrecht, E., Berghmans, D., Van der Linden, R.A.M.: 2009, Automated LASCO CME catalog for Solar Cycle 23: Are CMEs scale invariant? Astrophys. J. 691, 1222. DOI. ADS.

Rollett, T., Möstl, C., Temmer, M., Frahm, R.A., Davies, J.A., Veronig, A.M., Vršnak, B., Amerstorfer, U.V., Farrugia, C.J., Žic, T., Zhang, T.L.: 2014, Combined multipoint remote and in situ observations of the asymmetric evolution of a fast solar coronal mass ejection. Astrophys. J. Lett. 790, L6. DOI. ADS.

Rouillard, A.P., Davies, J.A., Forsyth, R.J., Rees, A., Davis, C.J., Harrison, R.A., Lockwood, M., Bewsher, D., Crothers, S.R., Eyles, C.J., Hapgood, M., Perry, C.H.: 2008, First imaging of corotating interaction regions using the STEREO spacecraft. Geophys. Res. Lett. 35, L10110. DOI. ADS.

Sachdeva, N., Subramanian, P., Colaninno, R., Vourlidas, A.: 2015, CME propagation: Where does aerodynamic drag 'take over'? Astrophys. J. 809, 158. DOI. ADS.

Sachdeva, N., Subramanian, P., Vourlidas, A., Bothmer, V.: 2017, CME dynamics using STEREO and LASCO observations: The relative importance of Lorentz forces and solar wind drag. Solar Phys. 292, 118. DOI. ADS.

Savani, N.P., Rouillard, A.P., Davies, J.A., Owens, M.J., Forsyth, R.J., Davis, C.J., Harrison, R.A.: 2009, The radial width of a Coronal Mass Ejection between 0.1 and 0.4 AU estimated from the Heliospheric Imager on STEREO. Ann. Geophys. 27, 4349. DOI. ADS.

Savani, N.P., Davies, J.A., Davis, C.J., Shiota, D., Rouillard, A.P., Owens, M.J., Kusano, K., Bothmer, V., Bamford, S.P., Lintott, C.J., Smith, A.: 2012, Observational tracking of the 2D structure of coronal mass ejections between the Sun and 1 AU. Solar Phys. 279, 517. DOI. ADS.

Sheeley, N.R. Jr., Michels, D.J., Howard, R.A., Koomen, M.J.: 1980a, Initial observations with the SOLWIND coronagraph. Astrophys. J. 237, L99. DOI. ADS.

Sheeley, N.R. Jr., Howard, R.A., Michels, D.J., Koomen, M.J.: 1980b, Solar observations with a new Earthorbiting coronagraph. In: Dryer, M., Tandberg-Hanssen, E. (eds.) Solar and Interplanetary Dynamics, IAU Symposium 91, 55. ADS.

Sheeley, N.R. Jr., Herbst, A.D., Palatchi, C.A., Wang, Y.-M., Howard, R.A., Moses, J.D., Vourlidas, A., Newmark, J.S., Socker, D.G., Plunkett, S.P., Korendyke, C.M., Burlaga, L.F., Davila, J.M., Thompson, W.T., St. Cyr, O.C., Harrison, R.A., Davis, C.J., Eyles, C.J., Halain, J.P., Wang, D., Rich, N.B., Battams, K., Esfandiari, E., Stenborg, G.: 2008, Heliospheric images of the solar wind at Earth. Astrophys. J. 675, 853. DOI. ADS.

St. Cyr, O.C., Burkepile, J.T., Hundhausen, A.J., Lecinski, A.R.: 1999, A comparison of ground-based and spacecraft observations of coronal mass ejections from 1980-1989. J. Geophys. Res. 104, 12493. DOI. ADS.

St. Cyr, O.C., Plunkett, S.P., Michels, D.J., Paswaters, S.E., Koomen, M.J., Simnett, G.M., Thompson, B.J., Gurman, J.B., Schwenn, R., Webb, D.F., Hildner, E., Lamy, P.L.: 2000, Properties of coronal mass ejections: SOHO LASCO observations from January 1996 to June 1998. J. Geophys. Res. 105, 18169. DOI. ADS.

St. Cyr, O.C., Flint, Q.A., Xie, H., Webb, D.F., Burkepile, J.T., Lecinski, A.R., Quirk, C., Stanger, A.L.: 2015, MLSO Mark III K-Coronameter observations of the CME rate from 1989 - 1996. Solar Phys. 290, 2951. DOI. ADS.

Tappin, S.J., Howard, T.A.: 2009, Interplanetary coronal mass ejections observed in the heliosphere: 1 . Review of theory. Space Sci. Rev. 147, 31. DOI. ADS. 
Tappin, S.J., Howard, T.A., Hampson, M.M., Thompson, R.N., Burns, C.E.: 2012, On the autonomous detection of coronal mass ejections in heliospheric imager data. J. Geophys. Res. 117, A05103. DOI. ADS.

Temmer, M., Veronig, A.M., Peinhart, V., Vršnak, B.: 2014, Asymmetry in the CME-CME interaction process for the events from 2011 February $14-15$. Astrophys. J. 785, 85. DOI. ADS.

Tousey, R., Howard, R.A., Koomen, M.J.: 1974, The frequency and nature of coronal transient events observed by OSO-7. Bull. Am. Astron. Soc. 6, 295. ADS.

van de Hulst, H.C.: 1950, The electron density of the solar corona. Bull. Astron. Inst. Neth. 11, 135.

Vourlidas, A., Balmaceda, L.A., Stenborg, G., Lago, A.D.: 2017, Multi-viewpoint coronal mass ejection catalog based on STEREO COR2 observations. Astrophys. J. 838(2), 141. DOI.

Webb, D.F., Howard, R.A.: 1994, The solar cycle variation of coronal mass ejections and the solar wind mass flux. J. Geophys. Res. 99, 4201. DOI. ADS.

Webb, D.F., Howard, T.A.: 2012, Coronal mass ejections: Observations. Liv. Rev. Solar Phys. 9, 3. DOI.

Webb, D.F., Mizuno, D.R., Buffington, A., Cooke, M.P., Eyles, C.J., Fry, C.D., Gentile, L.C., Hick, P.P., Holladay, P.E., Howard, T.A., Hewitt, J.G., Jackson, B.V., Johnston, J.C., Kuchar, T.A., Mozer, J.B., Price, S., Radick, R.R., Simnett, G.M., Tappin, S.J.: 2006, Solar Mass Ejection Imager (SMEI) observations of coronal mass ejections (CMEs) in the heliosphere. J. Geophys. Res. 111, A12101. DOI. ADS.

Yashiro, S., Michalek, G., Gopalswamy, N.: 2008, A comparison of coronal mass ejections identified by manual and automatic methods. Ann. Geophys. 26, 3103. DOI. ADS.

Yashiro, S., Gopalswamy, N., Michalek, G., St. Cyr, O.C., Plunkett, S.P., Rich, N.B., Howard, R.A.: 2004, A catalog of white light coronal mass ejections observed by the SOHO spacecraft. J. Geophys. Res. Space Phys. 109, A07105. DOI. ADS.

Yurchyshyn, V., Yashiro, S., Abramenko, V., Wang, H., Gopalswamy, N.: 2005, Statistical distributions of speeds of coronal mass ejections. Astrophys. J. 619, 599. DOI. ADS.

Zhang, J., Dere, K.P.: 2006, A statistical study of main and residual accelerations of coronal mass ejections. Astrophys. J. 649, 1100. DOI. ADS.

Zhang, J., Dere, K.P., Howard, R.A., Vourlidas, A.: 2004, A study of the kinematic evolution of coronal mass ejections. Astrophys. J. 604, 420. DOI. ADS.

Zhang, J., Richardson, I.G., Webb, D.F., Gopalswamy, N., Huttunen, E., Kasper, J.C., Nitta, N.V., Poomvises, W., Thompson, B.J., Wu, C.-C., Yashiro, S., Zhukov, A.N.: 2007, Solar and interplanetary sources of major geomagnetic storms (Dst $\leq-100 \mathrm{nt}$ ) during 1996-2005. J. Geophys. Res. 112(A10), A10102. DOI. 